Pacific Journal of Mathematics

UNIFORM NEIGHBORHOOD RETRACTS 


\title{
UNIFORM NEIGHBORHOOD RETRACTS
}

\author{
J. R. ISBELL
}

Introduction. This paper is a systematic investigation of a part of the mapping theory of uniform spaces. The central concept through most of the paper is that of an $A N R U$ as defined in [15]; that is, a uniform space $X$ which, whenever it is embedded in a uniform space $Y$, is a uniformly continuous retract of a uniform neighborhood of itself. Roughly, the first third of the paper treats the construction of $A N R U$ 's; the middle part treats necessary conditions for $A N R U$ 's and the last part treats some uses of $A N R U$ 's.

In compact spaces (and to a great extent in paracompact spaces) there is a well-developed theory analyzing the general space $X$ in terms of its mappings into polyhedra; in several strong senses, there are sufficiently many mappings into polyhedra. There are enough mappings even into a closed interval to determine the topology. There is also the program proposed by M. H. Stone [29] of analyzing $X$ in terms of mappings of Boolean spaces onto $X$; this is sufficient in principle, since every compact space is a quotient space of a Boolean space, but it seems to be very difficult to work out and in any case it cannot generalize much beyond the compact spaces. Note that the polyhedra are suitable for mapping into; they are $A N R$ 's, and a large (sufficiently large) class of them are $A R$ 's or injective objects. Gleason has shown [9] that a large class of Boolean spaces are projective objects in the category of compact spaces, but there remains the fundamental difficulty that the Boolean spaces themselves are not well known.

Among uniform spaces, finite-dimensional polyhedra are known to be $A N R U$ 's [15]; but there are not nearly enough of them. The basic difficulty here is that there are not enough mappings of uniform space $X$ into the real line, in the sense that knowing all of them does not suffice to determine the uniformity of $X$. The simple observation which generates Part $I$ of this paper is that if we know which real-valued functions on $X$ are uniformly continuous, and which families of real-valued functions on $X$ are equiuniformly continuous, this does suffice to determine the uniformity of $X$. Restated: every uniform space can be embedded in a product of spaces $U\left(D_{\alpha}, R\right)$ of all real-valued (uniformly continuous) functions on discrete spaces $D_{\alpha}$. As it happens, such a product space need not be an $A N R U$; but this can be changed by using a closed interval $I$ in place of $R$.

Section 1 gives the argument just sketched to prove that every

Received February 9, 1960. Research supported in part by a National Science Founda. tion Contract and in part by Office of Naval Recearch Contract Nonr-1100(12). 
uniform space can be embedded in an injective uniform space, and uses this to deduce simplified proofs of some known results. The other two sections of Part I pursue the same ideas in two different directions, as follows.

One can prove that a function space $U(D, I)$ is injective, when $D$ is discrete and $I=[0,1]$, by using Katětov's theorem [18] that $I$ is injective and observing that there is a natural one-to-one correspondence between mappings $X \rightarrow U(D, I)$ and mappings of the product space $X \times D$ into $I$. This depends on discreteness of $D$. In general, mappings $X \rightarrow U(Y, Z)$ correspond to mappings of $X * Y$ into $Z$, where $X * Y$ is a space which we call the semi-uniform product. We find a complicated description of $X * Y$, which simplifies considerably in case $Y$ is metric. The description shows that if $Y$ is metric, and $A$ is a subspace of $X$, then $A * Y$ is a subspace of $X * Y$. It follows that if (further) $Z$ is an $A N R U$, then $U(Y, Z)$ is also an $A N R U$.

Since $R$ is an $A N R U$, we have so far a large class of spaces of real-valued functions which are $A N R U$ 's. It follows easily that the Banach space of all bounded uniformly continuous real functions on a metric space is an $A N R U$. A quite different argument, depending on the space of all closed convex subsets, shows that every uniformly convex Banach space is an $A N R U$.

This concludes Part I. I do not have any examples to show necessity of the special hypotheses in $\S \S 2$ and 3 .

Part II again consists of a first section containing most of the ideas and two more sections working out some details. The approach is to deduce consequences from the hypothesis " $X$ is an $A N R U$ " until enough are in hand to prove a converse. For this I need three consequences: the homotopy extension lemma [15], a strong form of local contractibility, and the following generalization of a lemma of Hahn [11] for real-valued functions. For each uniform covering $\mathscr{U}$ of $X$ there is a finer uniform covering $\mathscr{y}^{\prime}$ such that for any uniform space $W$ and any function $f$ defined on $W$, with values in $X$, such that $f^{-1}(\mathscr{V})$ is a covering of $W$, there exists a uniformly continous function $g: W \rightarrow X$ within $\mathscr{U}$ of $f$.

The strong local contractibility property just mentioned is that every function space $U(W, X)$ is uniformly locally arcwise connected. It is cheering that $A N R U$ 's have this property; but for the characterization it would be desirable to make do with a local contractibility property which refers only to contraction of subsets. (The excuse for using the term for the stronger property is that it refers to contraction of "singular" subsets.) I do not know whether this is possible in general. It is in case every uniform covering of $X$ has a finite-dimensional uniform refinement.

Section 5 gives some simple consequences of local contractibility 
which lead to the conclusion that a locally compact $A N R U$ must be uniformly locally compact, and if it is injective (an $A R U$ ) it must be compact. This improves the known result [15] that an injective uniform space cannot be homeomorphic with the real line. Assuming the continuum hypothesis, we can go on to show that an $A N R U$ which is homeomorphic with the line must be uniformly equivalent with the line in the usual metric.

What is needed for the last result is not the whole continuum hypothesis, but the hypothesis that $2^{\boldsymbol{N}^{0}}<\boldsymbol{\aleph}_{\omega}$. The relevant lemma is that if $X$ is an $A N R U$ which is topologically separable metrizable, then the smallest cardinal number of a basis of uniform coverings of $X$ is a countable sum of smaller cardinals.

The last theorem in Part II is a topological analogue of the preceding: a compact $A N R$ of covering dimension 1 is metrizable. There are a number of open questions concerning higher-dimensional analogues of this and of the theorem about the line.

Part III is more mixed. The results of Section 7 concern inverse limits, $A N R U$ 's, and uniform complexes. For example, every complete space is an inverse limit of $A N R U^{\prime}$ 's. Those which are inverse limits of finite-dimensional complexes are determined. Section 8 is a detailed study of the known property (in $A N R$ 's and injective objects) of the category of uniformisable spaces and continuous functions. The main topic is intrinsically extensible mappings $f: A \rightarrow B$ in this category, defined by the condition that whenever $A$ is embedded as a closed subspace of $X, B$ can be embedded in some space $Y$ so that $f$ has a continuous extension over $X$ with values in $Y$. (In uniform spaces every uniformly continuous $f: A \rightarrow B$ has the analogous property.) Broadly, there are two kinds of intrinsically extensible mappings:

(a) the proper mappings and

(b) the mappings converging on the filter of complements of compact subsets of $A$ to a unique limit in $B$. Not even all the proper mappings are intrinsically extensible, except in locally compact spaces. More details are given.

Section 9 gathers up the results on mappings into $A N R U$ 's and into polyhedra, together with the corresponding results for compact spaces, in the context of the theory of adequacy in general categories. The general definitions of right and left adequacy [17] will be given in the body of the paper. The proposition that a class $K$ of objects of a category is right adequate is a precise way of saying that every object has sufficiently many mappings into objects of $K$. It is known [17] that for compact spaces, a square (2-cell) is right adequate, but a closed interval is not. ANRU's turn out to be right adequate for complete nniform spaces; 2-dimensional polyhedra (with uncountably many simplexes) 
are right adequate for a very large category of complete spaces. The results on left adequancy are much fuller and substantially less interesting.

I am indebted to H. H. Corson and to D. L. Johnson for several conversations on some of the questions examined here.

\section{Construction of ANRU's}

1. Function spaces. The reader should be acquainted with [15], or at least acquainted with the description of a uniformity as a family of coverings (the uniform coverings) and prepared for references to [15] and related papers. Here the term mapping, unqualified, will mean a uniformly continous mapping. Subspace means a uniform subspace, not necessarily closed. Recall that a mapping defined on a subspace with values in a complete space has a unique extension over the closure of the subspace. If $S$ is a subspace of $X$, a neighborhood $U$ of $S$ is a uniform neighborhood if it contains the star of $S$ with respect to some uniform covering of $X$.

We define a space $Y$ to be an extension space (neighborhood extension space) provided whenever $A \subset X$ and $f: A \rightarrow Y$ is a mapping, $f$ can be extended to a mapping of $X$ into $Y$ (to a mapping defined on some uniform neighborhood of $A$ ). It is shown in [15] that these properties are equivalent to the apparently weaker properties defined by setting $A=Y$; the space are called respectively $A R U$ 's and $A N R U$ 's. We shall use the new terms only for a few paragraphs, which will include a new proof of the equivalence. This depends on the known results that a closed interval $I$ is an extension space [18] and that every uniform space is a subspace of a product of spaces of functions with values in $I$. For the latter, combine the following more familiar results:

(a) every uniform space is a subspace of a product of metric spaces;

(b) a metric space with distance $d$ is uniformly equivalent to the same space with distance $\min (d, 1)$;

(c) a metric space $M$ with bounded distance $d$ is isometrically embedded in the Banach space of all bounded real functions on the set $M$ by sending each point $x_{0}$ to the function $d\left(x_{0}, x\right)$. Observe that the distance function on the Banach function space, $\|f-g\|=\sup |f(x)-g(x)|$, induces the usual uniformity of uniform convergence on the function space. Also, we shall later want to use the fact that an unbounded metric space $M$ can still be isometrically embedded in a function space [20].

A family $\left\{f_{\lambda} \mid \lambda \in L\right\}$ of functions on a set $A$ into a set $Y$ corresponds naturally to either of two single functions: the function $f^{*}$ on $A \times L$ into $Y$ defined by $f^{*}(a, \lambda)=f_{\lambda}(\alpha)$, or the function $f$ on $A$ into 
$Y^{L}$ defined by $f(a)(\lambda)=f_{\lambda}(a)$. We note

1.1. If $A$ and $Y$ are uniform spaces, $\left\{f_{\lambda}\right\}$ a family of functions on $A$ into $Y$ indexed by a discrete uniform space $L$ of indices, then the following conditions are equivalent.

(a) $\left\{f_{\lambda}\right\}$ is equiuniformly continuous.

(b) The corresponding function $f^{*}: A \times L \rightarrow Y$ is uniformly continuous.

(c) The corresponding function $f$ maps $A$ uniformly continuously into the function space $Y^{L}$.

The proof is immediate from the definitions of product and function space. We reserve the notation $Y^{L}$ for the set of all functions; when $X$ and $Y$ are uniform spaces, $U(X, Y)$ will denote the function space of all uniformly continuous functions on $X$ to $Y$.

1.2. THEOREM. Every equiuniformly continuous family of mappings of a subspace $A$ of a uniform space $X$ into a [neighborhood] extension space $Y$ can be extended to an equiuniformly continuous family of mappings of $X$ [of some fixed uniform neighborhood of $A$ in $X]$ into $Y$. If $Y$ is a [neighborhood] extension space then so is the space of all functions on any index set into $Y$.

Proof. The family $\left\{f_{\lambda} \mid \lambda \in L\right\}, f_{\lambda}: A \rightarrow Y$, or the single mapping $f: A \rightarrow U(L, Y)$, corresponds to $f^{*}: A \times L \rightarrow Y$. Since $A \times L$ is a subspace of $X \times L$, there is a uniformly continuous extension $g^{*}$ of $f^{*}$ defined on $X \times L$ [defined on a product neighborhood of $A \times L$ ]. Then $g^{*}$ induces an equiuniformly continuous family $\left\{g_{\lambda}\right\}$ extending $\left\{f_{\lambda}\right\}$, and a mapping $g$ extending $f$.

1.3. Corollary. The unit ball of the Banach space of all bounded real functions on any set is an extension space; every uniform space can be embedded in an extension space; every $A R U$ is an extension space.

Proof. The unit ball of the Banach space is the set of all functions with values in $I=[-1,1]$. Since every product of extension spaces is an extension space (by extending coordinatewise), the second assertion follows from the first; and the third follows from the second.

The last part of 1.3 is proved in [15], by different argument We recover also the following propositions, proved in [15]:

(a) every bounded uniformly continuous pseudometric on a subspace of a uniform space can be extended;

(b) if the bounded metric space $X$ is a uniformly continuous retract of every space containing it isometrically, then $X$ is an $A R U$. Similarly, 
of course, for $A N R U$ 's.

The corresponding propositions for unbounded metrics and pseudometrics are usually false, as is shown by the metric $\left|m^{3}-n^{3}\right|$ on the subspace of integers in the real line. For neighborhood extension and retraction, though, boundedness is not needed. This follows from 1.2 and the facts that the real line is an $A N R U$ [15] and that every metric space can be isometrically embedded in a Banach space of bounded real functions [20]. It is not stated in [15] that the real line is an $A N R U$; what is stated is that any finite-dimensional simplicial complex, metrized by maximum difference of bary centric coordinates (such a simplicial complex is called a uniform complex), is an $A N R U$. One easily verifies that any triangulation of the line yields a uniform complex uniformly equivalent to the line with the usual metric. But let us note a different proof, useful also for some other metric spaces.

1.4. The real line is an ANRU. Hence the Banach space of all bounded real functions on any set is an ANRU; every uniformly continuous pseudometric on a subspace of a uniform space can be extended over a uniform neighborhood; and for a metric space to be an ANRU, it suffices that it be a retract of a uniform noighborhood in every space containing it isometrically.

Proof. Let the line $R$, metrized by $\min (|x-y|, 4)$, be embedded in any metric $A R U, M$. It suffices to show that $R$ is a retract of its 1-neighborhood in $M$. For each $x$ in $M$, let $\lambda(x)$ be the distance of $x$ from $R$. For each $t$ in $(0,1)$, let $f(x, t)=\sup \{r \in R \mid d(x, r) \leqq(1+t) \lambda(x)\}$. If $\lambda(x) \leqq 1$, then $f(x, t)$ exists for each $t<1$, and for all $\varepsilon$ and $t$ in $(0,1)$, $3+f(x, \varepsilon)>f(x, t)$. Then $f(x, t)$ is a bounded monotonic function of $t$, and the Riemann integral $r(x)=\int f(x, t) d t$ exists. Evidently the function $r$ is a retraction upon $R$. To verify that $r$ is uniformly continuous, consider any positive $\delta$ such that $8 \delta+4 \delta^{2}<1$, and consider any two points, $x, y$, of $M$, within distance 1 of $R$ and $\delta^{2}$ of each other. In case either $x$ or $y$ is within $\delta$ of $R$, then both are within $\partial+\delta^{2}$ of $R$, and computaion shows that $d(r(x), r(y))<4 \delta+4 \delta^{2}$. In the contrary case, $\lambda(x)<\lambda(y)+\delta^{2}<(1+\delta) \lambda(y)$; then any point of $R$ within $(1+t) \lambda(x)$ of $x$ is within $(1+t+3 \delta) \lambda(y)$ of $y$, for $t<1$. For $t<1-3 \delta, f(y, t+3 \delta) \geqq$ $f(x, t)$; since also $f(y, t) \geqq f(x, s)-3-\delta^{2}$ for all $s$ and $t$ in $(0,1)$, we have $r(y)>r(x)-12 \delta$. Similarly $r(x)>r(y)-12 \delta$. Combining the cases, $d(r(x), r(y))$ cannot exceed $12 \delta$, and $r$ is uniformly continuous.

It follows by 1.2 that the function space $U(L, R)$ of all functions on a set $L$ to $R$ is an $A N R U$. In $U(L, R)$, the Banach subspace consisting of bounded functions is a uniform neighborhood of itself; hence it is also an $A N R U$. Then the remaining assertions are obvious. 
The main purpose of this section, the exhibition of sufficiently many $A R U$ 's and $A N R U$ 's for certain uses, is accomplished. We have also reviewed several of the basis facts from [15]. Let us recall further that every $A N R U$ is complete. (There are incomplete metric spaces $X$, e.g. $(0,1)$, which are retracts of all metric spaces containing $X$ isometrically as a closed subset.) Finally, for mappings into $A N R U$ 's, the homotopy extension lemma is valid [15], and in particular, every $A N R U$ which is contractible by a uniformly continuous homotopy (uniformly contractible) is an $A R U$.

2. Semi-uniform products. For any two uniform spaces, $A, B$ (in that order), we define a semi-uniform function $h$ on the Cartesian product set $A \times B$ into a uniform space $Y$ as a function such that the formula $h^{*}(a)(b)=h(a, b)$ defines a uniformly continuous mapping $h^{*}: A \rightarrow U(B, Y)$. It is almost obvious that the semi-uniform functions are precisely the uniformly continuous functions relative to a suitable uniformity on the product set; but this seems to require proof, and it is convenient to present the argument as follows. If we identify isometric pairs of metric spaces, there are only a limited number of functions mapping $A \times B$ onto a metric space. The semi-uniform product $A^{*} B$ is defined as the set $A \times B$ with the weak uniformity induced by all semi-uniform functions onto metric spaces. Observe that $A^{*} B$ is certainly a uniform space with a uniformity finer than the product uniformity (homeomorphic, however).

2.1. For any uniform spaces $A, B, Y$, the uniformly continuous functions on $A^{*} B$ into $Y$ are precisely the semi-uniform funstions on $A \times B$ into $Y$.

Proof. Suppose $h: A \times B \rightarrow Y$ is semi-uniform. Embed $Y$ in a product of metric spaces $M_{\alpha}$. For $h: A^{*} B \rightarrow Y$ to be uniformly continuous, it sufficies that each coordinate $h_{\alpha}: A^{*} B \rightarrow M_{\alpha}$ is uniformly continuous; and for this it suffices that $h_{\alpha}$ is semi-uniform. First, for each $a$ in $A$, the function $h_{*}^{*}(a): B \rightarrow M_{\alpha}$ is the composition of $h^{*}(a): B \rightarrow Y$ with coordinate projection $Y \rightarrow M_{\alpha}$; hence $h_{\alpha}^{*}(a) \in U\left(B, M_{\alpha}\right)$. Second, for each $\varepsilon>0$, there is a uniform covering $\mathscr{U}$ of $A$ such that for $a$ and $a^{\prime}$ in a single element of $\mathscr{L}$, for all $b, h_{\alpha}^{*}(a)(b)$ and $h_{\alpha}^{*}\left(a^{\prime}\right)(b)$ are within $\varepsilon$ of each other, since the subsets of $Y$ having $\alpha$ th coordinate projections of diameter $<\varepsilon$ form a uniform covering and $h^{*}$ is uniformly continuous. Thus $h$ is uniformly continuous.

Conversely, suppose $h$ is uniformly continuous on $A^{*} B$ into $Y$. By definition $A^{*} B$ is a subspace of a product of metric spaces $X_{\beta}$, with the coordinate projections $\pi_{\beta}$ semi-uniform on $A \times B$; that is, the projections correspond to uniformly continuous mappings $p_{\beta}: A \rightarrow U\left(B, X_{\beta}\right)$. We wish to prove $h$ is semi-uniform. First, for each $a$ in $A$, consider $h^{*}(a): B \rightarrow Y$. For any uniform covering $\mathscr{Y}$ of $Y$, there are finitely many 
indices $\beta_{1}, \cdots, \beta_{n}$ and a positive number $\varepsilon$ such that if $d\left(\pi_{\beta}(a, b), \pi_{\beta}\left(a, b^{\prime}\right)\right)<\varepsilon$ for $\beta=\beta_{1}, \cdots, \beta_{n}$, then $h(a, b)$ and $h\left(a, b^{\prime}\right)$ are in a single element of \%. Since each $p_{\beta}(A) \subset U\left(B, X_{\beta}\right)$, it follows that $h^{*}(a)$ is uniformly continuous. Finally consider $h^{*}: A \rightarrow U(B, Y)$. Again, since $h$ is uniformly continuous, for each $\mathscr{V}^{-}$we have $\beta_{1}, \cdots, \beta_{n}$ and $\varepsilon$ such that if $d\left(\pi_{\beta}(a, b), \pi_{\beta}\left(a^{\prime}, b\right)\right)<\varepsilon$ for these indices $\beta$, then $h(a, b)$ and $h\left(a^{\prime}, b\right)$ are near within $\mathscr{V}^{\prime}$; since all $p_{\beta}$ are uniformly continuous, there is a uniform covering $\mathscr{W}$ of $A$ such that this condition is satisfied when $a$ and $a^{\prime}$ are near within $\mathscr{W}$; and $h^{*}$ is uniformly continuous.

2.2. Every uniform covering of $A^{*} B$ has a refinement of the form $\left\{U_{\alpha} \times W_{\beta}^{\alpha}\right\}$, where $\left\{U_{\alpha}\right\}$ is a uniform covering of $A$ and for each $\alpha$, $\left\{W_{\beta}^{\alpha}\right\}$ is a uniform covering of $B$.

Proof. Every uniform covering has a uniformly continuous pseudometric subordinated to it; in short, there is a semi-uniform mapping $h$ into a metric space $M$ such that the given covering is refined by the inverse images of sets of diameter 1 . Now $h^{*}$ is uniformly continuous on $A$ into the metric function space $U(B, M)$; thus there is a uniform covering $\mathscr{V}^{*}$ of $A$, the diameters of the $h^{*}$-images of whose elements are at most $1 / 3$. For each $a$ in $A$, there is a uniform covering $\left\{W_{\beta}^{\alpha}\right\}$ of $B$ such that the diameters of the images of its elements under $h^{*}(a)$ are at most $1 / 3$. For each $a$, let $U_{a}$ be the star of $a$ with respect to \%. Then for any two points $(e, x),(f, y)$, of $A^{*} B$, both in a set $U_{a} \times W_{\beta}^{\alpha}$, $d(h(e, x), h(f, y)) \leqq d(h(e, x), h(a, x))+d(h(a, x), h(a, y))+d(h(a, y), h(f, y)) \leqq 1$, and the proof is complete.

On the other hand, a covering of the form described in 2.2 will be uniform on $A^{*} B$ if we can subordinate to it a suitable pseudometric. This requires precisely a descending sequence of coverings of the same form, with the $(n+1)$ st covering a star-refinement of the $n$th for all $n$. The problem closely resembles the problem of $[8,5.1]$, and by copying the arguments indicated there we shall construct such a sequence in case either $A$ has a basis of $\sigma$-discrete uniform coverings or $B$ is metric. In these cases, if $S$ is a subspace of $A, S^{*} B$ is a subspace of $A^{*} B$ (in the induced uniformity), since a covering of $S \times B$ of the form $\left\{U_{\alpha} \times W_{\beta}^{\alpha}\right\}$ can be extended to $\left\{V_{\alpha} \times W_{\beta}^{\alpha}\right\}$ on $A \times B$ by setting $V_{\alpha}=$ $U_{a} \cup(A-S)$. Whether this is valid for arbitrary $A$ and $B$ remains an open question.

Recall that a normal sequence of coverings is a sequence of coverings $\mathscr{Z}_{n}$ such that each $\mathscr{U}_{n+1}$ is a star-refinement of $\mathscr{U}_{n}$. For any normal sequence there is a pseudometric $d$ such that every element of the $n$th covering has $d$-diameter at most $2^{1-n}$, and every set of $d$-diameter $2^{-n}$ is contained in some element of the $n$th covering [14, proof of 1.0]. Then one readily verifies 
2.3. The uniform coverings of $A^{*} B$ are precisely those coverings which can be refined by normal sequences in which each term has a refinement of the form $\left\{U_{\alpha} \times W_{\beta}^{\alpha}\right\}$, where $\left\{U_{\alpha}\right\}$ is uniform on $A$ and for each $\alpha,\left\{W_{\beta}^{\alpha}\right\}$ is uniform on $B$.

\section{Note also}

2.4. Any uniform covering which is a countable union of disjoint collections has a uniform refinement which is $\sigma$-uniformly discrete.

Proof. Given $\left\{U_{\alpha}\right\}$, let $\left\{V_{\beta}\right\}$ be a star-refinement and let $U_{\alpha}^{\prime}$ be the union of those $V_{\beta}$ whose stars are contained in $U_{\alpha}$. Then for any disjoint family of $U_{\alpha}^{\prime}$ 's, the corresponding $U_{\alpha}^{\prime \prime}$ s are uniformly discrete.

2.5. Theorem. Suppose either that $B$ is a metric space or that $A$ has a basis of $\sigma$-discrete uniform coverings. Then every covering of $A \times B$ of the form $\left\{U_{\alpha} \times W_{\beta}^{\alpha}\right\}$, where $\left\{U_{\alpha}\right\}$ and each $\left\{W_{\beta}^{\alpha}\right\}$ are uniform, has a star-refinement of the same form and is therefore a uniform covering of $A \times B$. Hence for every subspace $S$ of $A, S^{*} B$ is a subspace of $A^{*} B$.

Proof. If $B$ is metric, there is a countable basis of uniform coverings $\mathscr{W}_{n}$; and we may suppose $\mathscr{W}_{n+1}$ is a star-refinment of $\mathscr{W}_{n}$, for each $n$. For each $\alpha$ choose $n=n(\alpha)$ such that $\mathscr{W}_{n}$ refines $\left\{W_{\beta}^{\alpha}\right\}$. Let $\left\{V_{\lambda}\right\}$ be a uniform covering of $A$ the star of whose star refines $\left\{U_{\alpha}\right\}$. For each $\lambda$ let $p(\lambda)$ be the least $i$ such that for some $U_{a}$ containing the second star of $V_{\lambda}, n(\alpha)=i$. Let $q=q(\lambda)=p(\lambda)+1$. Then the covering consisting of all sets $Z_{\lambda \mu}=V_{\lambda} \times Y_{\mu}^{q}$, where $q=q(\lambda)$ and $Y_{\mu}^{q} \in \mathscr{W}_{q}$, is a star-refinement of the given one. Specifically, the star of $Z_{\lambda \mu}$ is contained in any $U_{\alpha} \times W_{\beta}^{\alpha}$ satisfying

(i) $U_{\alpha}$ contains the star of $V_{\lambda}$, and subject to this condition, $n(\alpha)$ is a minimum;

(ii) $W_{\beta}^{\alpha}$ contains the star of $Y_{\mu}^{q}$ with respect to $\mathscr{W}_{n(\alpha)+1}$. (Since $n(\alpha) \leqq p(\lambda)$, (ii) can be satisfied.) For the proof, suppose $V_{\sigma} \times Y_{\tau}^{r}$ meets $Z_{\lambda \mu}$. $\quad V_{\sigma}$ meets $V_{\lambda}$ and is contained in $U_{\alpha}$. Clearly $q=q(\lambda) \geqq n(\alpha)+1$. As for $r$, it is $n(\gamma)+1$ for some $U_{\gamma}$ containing the second star of $V_{\sigma}$; this contains the star of $V_{\lambda}$, so that $n(\gamma) \geqq n(\alpha)$. Then $Y_{\tau}^{r}$ is contained in the star of $Y_{\mu}^{q}$ with respect to $\mathscr{W}_{n(\alpha)+1}$, which is contained in $W_{\beta}^{\alpha}$, and the proof for this case is complete.

Now suppose $A$ has a basis of $\sigma$-discrete coverings. Suppose in particular that $\left\{U_{\alpha}\right\}$ is re-indexed as $\left\{U_{n \alpha}\right\}$, where for fixed $n$ the different sets $U_{n a}$ are disjoint. Again let $\left\{V_{\lambda}\right\}$ be a uniform covering of $A$ the star of whose star refines $\left\{U_{n \alpha}\right\}$. For each $\lambda$ let $p(\lambda)$ be the least $n$ such that some $U_{n \alpha}$ contains the star of the star of $V_{\lambda}$. For each $n$ and $\alpha$, let $\mathscr{W}_{n \alpha}$ be a uniform covering of $B$ which is a star-refinement of $\left\{W_{\beta}^{n \alpha}\right\}$. For each $\lambda$, for each $i \leqq p(\lambda)$, there is at most one $\alpha$ such 
that $U_{i \alpha}$ contains $V_{\lambda}$; let $\mathscr{Y}_{\lambda}=\left\{Y_{\mu}^{\lambda}\right\}$ be a uniform covering of $B$ which is finer than all $\mathscr{W}_{i \alpha}$, for these $i$ and $\alpha$. Then $\left\{V_{\lambda} \times Y_{\mu}^{\lambda}\right\}$ is a starrefinement of $\left\{U_{n \alpha} \times W_{\beta}^{n \alpha}\right\}$, just as in the preceding case; we omit the details.

2.6. Corollary. If $B$ is a metric space, and $Y$ is an ANRU or $A R U$, then so is the function space $U(B, Y)$.

Proof. In this case the problem of extending $f: A \rightarrow U(B, Y)$ over $X \supset A$ is equivalent to the problem of extending $f^{*}: A^{*} B \rightarrow Y$ over $X^{*} B$. The assertion about $A R U$ 's follows at once; the assertion about $A N R U^{\prime}$ 's follows in view of 2.2 , since a uniform neighborhood of $A^{*} B$ must contain a product neighborhood.

The $\sigma$-discrete case in 2.5 tells us that even if $B$ is not metric, $U(B, Y)$ admits extensions for suitable $X \supset A$. Note that 2.4 is not a lemma for the proof of 2.5; it merely shows that whether we say " $\sigma$ discrete", " $\sigma$-disjoint", or " $\sigma$-uniformly discrete", we have the same hypothesis.

From 2.5 we can also conclude that if $C$ is metric, $B \subset C$, and $A$ is arbitrary, then $A^{*} B$ is a subspace of $A^{*} C$. One can deduce that for any $A R U, E$, there is a uniformly continuous mapping of $U(B, E)$ to $U(C, E)$ which takes each function to an extension of itself (taking $A=U(B, E))$. However, this does not seem to be the right approach to such simultaneous extension problems. The result just noted, for spaces of bounded real functions, follows easily from the arguments of McShane in [22]; also, for spaces of continuous functions, there are frequently continuous linear mappings of this sort. The main reference is Dugundji [5].

3. Banach spaces. It seems likely that every Banach spaces is an $A N R U$. The Banach spaces are certainly not $A R U$ 's because they are not uniformly contractible. Evidently when a Banach space $E$ is an $A N R U$, every bounded convex body in $E$ is a uniformly contractible retract of $E$ and thus an $A R U$. The affirmative results are summarized in

3.1. THEOREM. The following Banach spaces are ANRU's: the space $U^{*}(B, R)$ of bounded uniformly continuous real-valued functions on any metric space $B$; the injective Banach spaces, i.e., the injective objects of the category of Banach spaces and bounded linear transformations; and the uniformly convex Banach spaces. Moreover, each of these spaces is a uniformly continuous retract of any metric space containing it isometrically. 
Proof. We know immediately from 2.6 that $U^{*}(B, R)$ is an $A N R U$; however, we shall give a direct proof of the stronger result that it is a retract of any space containing it isometrically. From this the result on injective Banach spaces $E$ follows at once, for $E$ can be embedded as a closed linear subspace of $U^{*}(K, R)$, where $K$ is the unit ball of the conjugate space.

We begin with the observation

(a) The Banach space $F$ of all bounded real-valued functions on a set $B$ is a uniformly continuous retract of any metric space containing it isometrically.

This is proved just like 1.4 (we used a bounded metric on $R$ in 1.4 not for the retraction, but in order to appeal to Katetov's extension theorem for $I$ in completing the proof). If $F$ is contained in a metric space $M$ with distance $d$, define $\lambda(p)=d(p, F)$ for $p$ in $M ; h(p, t, b)=$ $\sup \{f(b) \mid f \in F, d(p, f) \leqq(1+t) \lambda(p)\}$ for $p \in M, t \in(0,1), b \in B$; and $r(p)(b)=$ $\int_{0}^{1} h(p, t, b) d t$. Just as before, $r$ is uniformly continuous.

(b) If the index set $B$ is a metric space, the subspace $U^{*}(B, R)$, is a uniformly continuous retract of $F$.

Let $d$ be an equivalent bounded metric on $B$. For $f$ in $F$, let $\lambda(f)$ be the distance of $f$ from the subspace $U^{*}$. If $\lambda(f) \neq 0$ then, since $f$ is within $3 \lambda(f) / 2$ of a uniformly continuous function, there is $\varepsilon>0$ such that the diameter in $R$ of the image under $f$ of any set of $d$-diameter $\varepsilon$ is at most $3 \lambda(f)$. Let $\mu(f)$ be the least upper bound of all such $\varepsilon$, the diameter of $B$ where the diameter of $f(B)$ does not exceed $3 \lambda(f)$, and 0 precisely on the subspace $U^{*}$. For $t$ in $[0,1], x$ in $B$, define $h(f, x, t)$ as the least upper bound of the set of all values $g(y)$ of functions $g$ satisfying $\|g-f\| \leqq t \lambda(f)$ at points $y$ satisfying $d(x, y) \leqq t \mu(g)$. Observe that $g(x) \leqq f(x)+t \lambda(f), g(y) \leqq g(x)+3 \lambda(g)$, and in paticular $f(x)+7 \lambda(f)$ is an upper bound for $h(f, x, t)$, while $f(x)$ is a lower bound. If $f$ is in $U^{*}$ then $h(f, x, t)=f(x)$ for all $x$. In any case $j(f, x)=\int h(f, x, t) d t$ is uniformly continuous in $x$; for if $f$ is not in $U^{*}$, then $\mu(f)>0$, and for $x$ and $y$ within $\delta \mu(f)$ of each other we have $|j(f, x)-j(f, y)|<$ $\delta\|f\|+7 \delta \lambda(f)$. Then a retraction $r$ may be defined by $r(f)(x)=j(f, x)$.

To verify uniform continuity of $r$, suppose $\|f-g\|<\delta^{2}<1$. If $\lambda(f)$ or $\lambda(g)$ is less than $\delta$, we have $r(f)$ within $7 \delta+7 \delta^{2}$ of $f$, similarly for $g$, and $\|r(f)-r(g)\|<14 \delta+15 \delta^{2}$. In the remaining case the smaller of $\lambda(f)$ and $\lambda(g)$ is $k \delta$ for some $k \geqq 1$. Then $(1+\delta / k) \lambda(f) \geqq \lambda(g)$ and symmetrically. It follows that $h(f, x, t+\delta / k) \geqq h(g, x, t)$ for $t<1-\delta / k$ and symmetrically. On the other hand, the difference between $h(f, x, u)$. and $h(g, x, v)$ for any $u$ and $v$ in $[0,1]$ does not exceed the supremum of the expressions $f^{\prime}(y)-g^{\prime}(z)=f^{\prime}(y)-f^{\prime}(x)+f^{\prime}(x)-f(x)+f(x)-g(x)+$ $g(x)-g^{\prime}(x)+g^{\prime}(x)-g^{\prime}(z)$, where $\left\|f^{\prime}-f\right\| \leqq \lambda(f)$, so that $\lambda\left(f^{\prime}\right) \leqq 2 \lambda(f)$, and $d(x, y) \leqq \mu\left(f^{\prime}\right)$, so that $\left|f^{\prime}(y)-f^{\prime}(x)\right| \leqq 3 \lambda\left(f^{\prime}\right) \leqq 6 \lambda(f)$, and similarly 
for $g^{\prime}$. Then $\|r(f)-r(g)\| \leqq \delta / k\left(7 \lambda(f)+\delta^{2}+7 \lambda(g)\right)<29 \delta^{2}$ in this case, and $r$ is uniformly continuous. This proves $(b)$.

From (a) and (b) we obtain the theorem for $U^{*}(B, R)=U^{*}$; for if $U^{*}$ is contained isometrically in $M$, let $N$ be the quotient space of $M \cup F$ obtained by identifying corresponding points of $U^{*}$ and defining the distance between a point $x$ of $M$ and a point $y$ of $F$ as $\inf \left\{d(x, z)+d(z, y) \mid z \in U^{*}\right\}$. From (a), this space can be retracted upon $F$, and if we then retract $F$ upon $U^{*}$ as in (b) we have in particular a retraction of $M$ upon $U^{*}$.

For the rest of the proof, we need

3.2. Lemma. For every Banach space $E$, the space $H(E)$ of bounded closed convex subsets, metrized by Hausdorff distance, is a uniformly continuous retract of any space containing it isometrically.

In 3.2 the empty set is not meant to be a point of $H(E)$. If it is adjoined as an isolated point then of course one obtains a disconnected $A N R U$. We remark also that the lemma extends to the space of all bounded closed sets, since taking each set to its convex hull is a uniformly continuous retraction. We prove next

(c) 3.2 implies 3.1.

We need a retraction $r$ assigning to each closed bounded convex set $S \in H(E)$ a point $r(S) \in E$. Let $r(S)$ be the unique point of $S$ whose norm is a minimum. From the definition of uniform convexity, for each positive $\varepsilon$ there is $\delta>0$ such that the midpoint of a segment of length $\varepsilon$ joining two points of norm 1 has norm at most $1-\delta$. Suppose $p$ has norm $n, q$ has norm $n(1+\theta)$ or less, and $\|p-q\| \geqq \varepsilon+\theta n$. By applying the definition to $(1 /(1+\theta)) q$ we find on the segment $p q$ a point of norm at most $n(1-\delta)(2+2 \theta) /(2+\theta)$. For sufficiently small $\theta$ (and $n \neq 0$ ) this is less than $n$. Let $\alpha$ be that small, and consider two sets $S, T$ (elements of $H(E)$ ) at distance at most $\alpha \varepsilon$. If either $S$ or $T$ has a point of norm $\varepsilon$ or less, evidently $\|r(S)-r(T)\| \leqq 2 \varepsilon+\alpha \varepsilon$. In the contrary case let $p$ denote the one of $r(S)$ and $r(T)$ with larger norm; if they are equal, choose either, and by relabeling if necessary, suppose $p=r(S),\|p\|=n$. There is a point $q$ of $S$ within $\alpha \varepsilon$ of $r(T)$. Then $\|q\| \leqq n+\alpha \varepsilon=n(1+\theta)$, where $\theta=\alpha(\varepsilon / n)$ is even smaller than $\alpha$. Since $S$ contains the segment from $p$ to $q$ and $p=r(S),\|p-q\|<$ $\varepsilon+\theta n=\varepsilon+\alpha \varepsilon$, and $\|r(S)-r(T)\|<\varepsilon+2 \alpha \varepsilon$. Thus $r$ is uniformly continuous.

Proof of 3.2. With $H(E)$ isometrically embedded in a metric space $X$, we shall define $r: X \rightarrow H(E)$ by $r(x)=\int_{0}^{1} h(x, t) d t$ with $h(x, t)=$ $\sup \{y \in H(E) \mid d(x, y) \leqq(1+t) d(x, H(E))\}$, just as before. The integration involved here is a fairly natural one, but it does not seem to have been treated in the literature. Perhaps one reason for this is that it is 
simplest to define a Riemann-type integral in this situation.

The order in $H(E)$ is the inclusion ordering.

Let $g$ be a monotonic function on the closed interval $[0,1]$ into the space of bounded closed convex subsets $H(E)$. For any finite partition $\pi$ of $[0,1]$ into subintervals with end points (in natural order) $p_{1}, \cdots, p_{n+1}$, with lengths $p_{i+1}-p_{i}=a_{i}$, we define the lower sum $L(\pi)$ in $H(E)$ to be the set of all points $\Sigma a_{i} x_{i}$ in $E$ with $x_{i} \in g\left(p_{i}\right)$ for $i=1, \cdots, n$. The upper sum $U(\pi)$ is the set of all $\Sigma a_{i} x_{i}$ with $x_{i} \in g\left(p_{i+1}\right)$ for all $i$. Evidently every upper sum contains every lower sum. Further, any $y$ in $E$ which is in all upper sums can be approximated to within any $\delta>0$ by an element of a lower sum. For this let $k=d(g(0), g(1))$, and let $\pi$ be a uniform partition of mesh $1 / n<\delta / k$. Express $y$ as $\Sigma(1 / n) x_{i}$ with $x_{i}$ in $g\left(p_{i+1}\right)$; then choose $x_{0}$ in $g(0)$ within $k$ of $x_{n}$, and observe that $\Sigma(1 / n) x_{i-1}$ is an element of $L(\pi)$ within $\delta$ of $y$.

Then for any such function $g$, the least upper bound of the lower sums coincides with the greatest lower bound of the upper sums, and will be called $\int g(t) d t$ or simply $I(g)$. Next let $g$ and $h$ be two such functions, let $k$ be a bound for all $d(g(s), h(t))$, and suppose $g(t+\delta) \geqq$ $h(t)$ for $t$ in $[0,1-\delta]$. Then the closed $k \delta$-neighborhood of $I(g)$ contains $I(h)$; we omit the routine check. In particular, if also $h(t+\delta) \geqq g(t)$, then $d(I(g), I(h)) \leqq k \delta$.

But this is all properties of the integral that we need. To get a function $h(x, t)$ defined for $t=0$, take $\sup \{y \in H(E) \mid d(x, y) \leqq$ $(2+t) d(x, H(E))\}$, and the proof goes as before.

REMARKs. The injective Banach spaces are a rather narrow class, including the spaces $F$ of all functions but not the spaces $U^{*}$ [4].

The problem whether a given Banach space $E$ is an $A N R U$ is equivalent to the problem whether it is a uniform neighborhood retract of $H(E)$.

The simplest Banach space for which the problem is open would seem to be the space $U(W, R)$, where $W$ is the space of countable ordinals. The sequence space $l_{1}$ is not covered by 3.1 , but it is easy to construct a retraction of $l_{\infty}$ upon it.

\section{Restrictions on $A N R U$ 's.}

4. General properties. An obvious necessary condition for a space to be an $A N R U$ is that it is topologically an $A N R$ in some sense. We shall give a precise form to this statement at the end of this section, and follow it up in $\S \S 5$ and 6 . For the present we are concerned with broad uniform properties. The notion of an $A N R U$ splits rather neatly into three parts. One of these, the homotopy extension lemma, will 
not be further examined here. Most of the difficulties seem to lie in the second "broad" property, local contractibility. First we take up the third property, one which has not attracted much separate attention in topological spaces but which is frequently used for the special case of polyhedra. Its first occurrence in the literature seems to be in a 1917 paper of Hahn [11], for real-valued functions on metric spaces; a generalization to real-valued functions on uniform spaces is given in [3]. We shall use that result: Any real-valued function $f$ on a uniform space $X$ such that the inverse image of the covering of the real line consisting of all intervals of length $\varepsilon$ is a uniform covering of $X$ can be approximated within $\varepsilon$ at every point of $X$ by a uniformly continuous function $g$.

4.1. (Hahn's lemma). For any ANRU Y, for each uniform covering $\mathscr{U}$ of $Y$, there is a uniform coveriug $\mathscr{Y}$ of $Y$ such that for any uniform space $X$ and any function $f$ defined on $X$ with values in $Y$, such that the inverse image of $\mathscr{Y}$ under $f$ is a uniform covering of $X$, there exists a uniformly continuous function $g: X \rightarrow Y$ within $\mathscr{U}$ of $f$.

Proof. Recall that $Y$ is a subspace, and therefore a uniform: neighborhood retract, of a product $P$ of spaces $F_{a}$ each of which is the space of all bounded functions on a set $D_{\alpha}$ to $R$. We prove first that the lemma is true if $Y=F_{\alpha}$. For each uniform covering $\mathscr{U}$ of the function space $F_{\alpha}$ there is $\varepsilon>0$ such that any two functions in $F_{\alpha}$ which are pointwise within $\varepsilon$ of each other are within $\mathscr{U}$ of each other. Let $\mathscr{y}$ be the covering of the metric function space $F_{a}$ consisting of all spheres of radius $\varepsilon / 2$. Let $f: X \rightarrow F_{\alpha}$ be any function under which the inverse image of $\mathscr{V}$ is uniform. Then $f^{*}: X \times D_{\alpha} \rightarrow R$ varies no more than $\varepsilon$ on any set $W \times\{d\}, W$ a member of $f^{-1}(\mathscr{V}), d$ a point of $D_{\alpha}$. Since $D_{a}$ is discrete, these sets form a uniform covering of the product space. From [3], there is a uniformly continuous function $g^{*}$ on $X \times D_{a}$ to $R$ within $\varepsilon$ of $f$. The corresponding function $g: X \rightarrow F_{\alpha}$ is then a uniformly continuous function $\mathscr{U}$ of $f$.

Now consider product spaces $P$ of factors $F_{\alpha}$. Each uniform covering $\mathscr{U}$ of $P$ is refined by a product of uniform coverings $\mathscr{U}_{\alpha}$ of $F_{\alpha}$, all but finitely many of which are the one-element coverings $\left\{F_{\alpha}\right\}$. To approximate a function $f: X \rightarrow P$ to within $\mathscr{U}$ it suffices to approximate each coordinate function $f_{\alpha}$ to within $\mathscr{U}_{\alpha}$, which we have just shown to be possible if the inverse image under $f_{\alpha}$ of a suitable covering $\mathscr{V}_{\alpha}$ is uniform. Only finitely many coordinates are restricted, and thus the lemma is true for $P$. Finally suppose $Y$ is a subspace of $P$ and a retract of a uniform neighborhood $U$, by a mapping $r: U \rightarrow Y$. One approximates $f: X \rightarrow Y$ by first taking an approximation $g$ with values in $P$, close enough so that $g(X) \subset U$, and then using $r g: X \rightarrow Y$. To have $r g$ 
within a specified covering $\mathscr{U}$ from $f$, we must have $g$ within $r^{-1}(\mathscr{U})$ from $f$. But $r^{-1}(\mathscr{C})$ is a uniform covering of $U$, and since $U$ is a uniform neighborhood, $r^{-1}(\mathscr{U})$ together with the set $P-Y$ forms a uniform covering $\mathscr{Q}^{\prime}$ of $P$. There is a covering $\mathscr{V}^{-\prime}$ of $P$ fine enough so that any $f: X \rightarrow P$ under which the inverse image of $\mathscr{V}^{\prime}$ is uniform can be approximated to within $\mathscr{U}^{\prime}$ by a uniformly continuous mapping $g$. If all values of $f$ are in $Y$, then all values of $g$ must be in $U$, and the proof is complete.

The proof of 4.1 shows, of course, that the class of spaces $Y$ for which Hahn's lemma is valid is closed under the constructions used there. One can show in a similar manner that the class is closed under formation of spaces $U(B, Y)$ at least for metric spaces $B$. The class is much larger than the class of $A N R U$ 's. For example, it is easy to prove that Hahn's Lemma remains valid for any compact totally disconnected space $Y$.

A uniform space is uniformly locally arcwise connected provided it has a basis of uniform coverings each of which consists of arcwise connected sets. The sense of the term "arcwise connected" is topological; since an arc is compact, there is no question about its uniform structure. Recall that arcwise connectedness is equivalent to pathwise connectedness. We define a space $Y$ to be locally contractible in the uniform sense, or $L C U$, provided for every uniform space $X$ the function space $U(X, Y)$ is uniformly locally arcwise connected.

The property just defined is not defined in terms of contractions, but it belongs to a family of properties commonly thought of as " $L C$ ", properties. We avoid the term "uniformly locally contractible", which is already in use for one or more weaker properties. We can deduce from $L C U$ the existence of local contractions in a very strong sense. To state this sense, consider a family $\left\{f_{\alpha}\right\}$ of mappings having different domains $X_{\alpha}$ and a common range $Y$. Relative to a space $X$ containing all $X_{\alpha}$ as subspaces, $\left\{f_{\alpha}\right\}$ will be called an equiuniformly continuous family if for each uniform covering $\mathscr{C}$ of $Y$ there is a uniform covering $\mathscr{Y}$ of $X$ whose restriction to each $X_{a}$ is a refinement of $f_{\alpha}^{-1}(\mathscr{U})$. A space $Y$ will be called $L C W U$ provided for each uniform covering $\mathscr{C}$ of $Y$ there exist a uniform covering $\mathscr{V}$ and an equiuniformly continuous family of homotopies $\left\{h_{V} \mid V \in \mathscr{Y}\right\}$, each $h_{V}$ contracting $V$ to a point within some $U \in \mathscr{\%}$. (In particular, $\mathscr{Y}$ is a refinement of \%. Equiuniform continuity here is with respect to $Y \times I$.)

4.2. Every ANRU is LCU and every LCU space is $L C W U$. For finite-dimensional compact metric spaces these concepts are equivalent.

Proof. The real line is obviously $L C U$; the function spaces $U(X, R)$ are sums of components which are uniformly equivalent to Banach spaces, 
and two functions $f$ and $g$ at finite distance are joined by a path of functions $t f+(1-t) g$. To prove from this that every $A N R U$ is $L C U$ is a simple exercise along the lines of 4.1 , which we leave to the reader. Now let $Y$ be an $L C U$ space. For each uniform covering $\mathscr{V}$ of $Y$ let $X\left(\mathscr{Y}^{-}\right)$be the uniform space defined as follows. The points of $X(\mathscr{Y})$ are the ordered pairs $(x, V)$, where $x \in V \in \mathscr{Y}$. For each $V \in \mathscr{Y}$, let $V^{\prime}(\mathscr{V})$, or $V^{\prime}$ for short, denote the subset of $X\left(\mathscr{V}^{-}\right)$consisting of all ordered pairs whose second member is $V$. A covering $\mathscr{U}^{\prime}$ of $X(\mathscr{V})$ is defined to be uniform provided there exists a uniform covering $\mathscr{U}$ of $Y$ such that for each $U \in \mathscr{U}^{\prime}$ and each $V \in \mathscr{Y},\{y \in Y \mid(y, V) \in U\}$ is a subset of some member of $\mathscr{U}$. Evidently $X(\mathscr{V})$ is a uniform space, the subsets $V^{\prime}$ form a uniformly discrete collection and a uniform covering, and the first coordinate mapping $\pi: X\left(\mathscr{Y}^{-}\right) \rightarrow Y$ is uniformly continuous. Let $X$ denote the sum of all $X\left(\mathscr{V}^{n}\right)$. (In a sum space, a covering is uniform if and only if its restriction to each summand is uniform.)

Since $Y$ is $L C U$, the function space $U(X, Y)$ is uniformly locally arcwise connected. Thus for each uniform covering $\mathscr{U}$ of $Y$ there is a uniform covering $\mathscr{Y}$ of $Y$ such that any two functions $h_{0}, h_{1}$, in $U(X, Y)$ within $\mathscr{V}$ of each other are joined by a path $h: I \rightarrow U(X, Y)$. lying within some element of $\mathscr{U}$. Let $h_{0}$ be the projection $\pi$; let $h_{1}$ coincide with $h_{0}$ except on the subspace $X\left(\mathscr{V}^{\prime}\right)$, where on each $V^{\prime} h_{1}$ has some constant value $p(V) \in V$. The corresponding path $h$ induces in turn a mapping $h^{*}: I^{*} X \rightarrow Y$. But $I^{*} X$ is $I \times X$ (this is essentially a well-known fact; at any rate it follows at once from 2.5). Then the homotopies $h_{V}$ obtained by restricting $h^{*}$ to the subsets $I \times V^{\prime}, V^{\prime}$ in $X\left(\mathscr{Y}^{\prime}\right)$, form the required equiuniformly continuous family of contractions.

Next, it is known [6] that for finite-dimensional metric spaces, locally contractible $\Rightarrow A N R$ for metric spaces. In the compact case, these properties are equivalent respectively to $L C W U$ and to $A N R U$. In fact, $L C W U \Rightarrow$ locally contractible in the (standard) sense of [6] for all uniform spaces. Since every compact metric space can be embedded in the Hilbert cube, a metric space which is an $A R U$, we conclude $A N R \Rightarrow A N R U$, as required.

I do not have an example of an $L C W U$ space which is not $L C U$. Borsuk has given [2] an example of a locally contractible compact metric space which is not an $A N R$. It does not seem likely that there is a useful characterization of ANRU's in terms of simpler properties. The following result may be called an instructive characterization.

4.3. Every LCU space satisfying Hahn's lemma and the homotopy extension lemma is an ANRU. If every uniform covering of $Y$ has a finite-dimensional uniform refinement, and $Y$ is $L C W U$ and satisfies the homotopy extension lemma, then $Y$ is an ANRU.

The proof will not be given in detail. For the first assertion, given 
$f: A \rightarrow Y$ and $A \subset X$, use the property $L C U$ first to get a uniform covering $\mathscr{U}$ of $Y$ such that any two mappings which are near of order $\mathscr{U}$ are homotopic. Then take a covering $\mathscr{Y}$ of $Y$ suitable for Hahn's lemma, so that a discontinuous function within $\mathscr{Y}$ of being uniformly continuous and within $\mathscr{Y}$ of $f$ can be approximated by a uniformly continuous function still within $\mathscr{Q}$ of $f$. Then take a sufficiently fine uniform covering $\left\{W_{\alpha}\right\}$ of $A$, shrink it to $\left\{R_{\alpha}\right\}$, and extend this to a uniform covering $\left\{S_{\alpha}\right\}$ of a uniform neighborhood $N$ of $A$ so that no two $S_{\alpha}$ 's meet unless the corresconding $W_{\alpha}$ 's meet. This can be done so that a function $g$ on $N$ to $Y$ assigning to each $p \in N$ an arbitrary $f(x)$, $x$ in some $S_{\alpha}$ containing $p$, can be approximated by uniformly continuous $h: N \rightarrow Y$ closely enough so that $h \mid A$ is homotopic to $f \mid A$, which will therefore be extensible over $N$.

The second part is more complicated. Given $f: A \rightarrow Y, A \subset X$, take a uniform covering $\mathscr{C}$ of $Y$ whose numbers are equiuniformly continuously contractible. Take a finer uniform covering which is a finite union of uniformly discrete subfamilies $\mathscr{Y}^{i}=\left\{V_{a}^{i}\right\}, i=1, \cdots, m$ (see $[15,2.2]$ ). Then the sets $W_{\alpha}^{i}=f^{-1}\left(V_{\alpha}^{i}\right)$ form a uniform covering of $A$ and for fixed $i,\left\{W_{\alpha}^{i}\right\}$ is uniformly discrete. Construct $\left\{S_{\alpha}^{i}\right\}=\mathscr{S}$ covering a uniform neighborhood $N$ of $A$ in $X$, as before. Then construct the space $Z$ of ordered pairs $(x, S), x \in S \in \mathscr{S}$, as in 4.2. By the contractions of sets containing the sets $W_{\alpha}^{i}$, the mapping $f \pi: \pi^{-1}(A) \rightarrow Y$ is homotopic to a mapping which is constant on each $\pi^{-1}\left(S_{\alpha}^{i}\right)$ and therefore extensible over $Z$. However, we need an extension $h$ which is of the form $g \pi$, where $g$ is a uniformly continuous extension of $f$ over $N$. This is easily constructed by means of functions $j_{1}, k_{1}, j_{2}, k_{2}, \cdots, j_{m}=h$, where $j_{1}$ is an extension of $f \pi$ over the subset of $Z$ consisting of $\pi^{-1}(A)$ and all $\left(x, S_{\alpha}^{\prime}\right)$, $k_{1}$ is the unique extension of $j_{1}$ over the union of $\pi^{-1}(A)$ and all $\pi^{-1}\left(S_{\alpha}^{\prime}\right)$ which has the form $l_{1} \pi$, and so on.

We conclude with

4.4. Every ARU [ANRU] is a [neighborhood] extension space for collectionwise normal spaces.

This is a trivial corollary of Dugundji's theorem [5] that every Banach space is an extension space for collectionwise normal spaces, since every $A N R U$ is complete and is thus a closed subspace of a product of Banach spaces.

5. Contractibility, metrizability. For $A R U$ 's, uniform contractibility has some immediate corollaries. These conclusions of course apply uniformly locally to $A N R U$ 's. A first corollary was noted in [15]: a uniformly contractible space $X$ is finitely chainable in the sense of Atsuji [1], i.e., for every uniform covering $\mathscr{Q}$ of $X$ there exist finitely many elements $U_{1}, \cdots, U_{m}$ of $\mathscr{U}$ and a finite cardinal $n$ such that every 
element of $\mathscr{\mathscr { C }}$ can be joined to one of $U_{1}, \cdots, U_{m}$, by a chain of $n$ or fewer successively intersecting elements of $\mathscr{U}$. Note that this property is preserved under uniformly continuous images and is therefore possessed "equi-uniformly locally" by $L C W U$ spaces. Since there is already a concept of chainable involving the existence of a basis of coverings which are in some sense chains, we suggest the alternative term uniformly bounded for Atsuji's concept. A good reason for the term was given already (essentially) by Atsuji.

5.1. (Atsuji). A uniform space is uniformly bounded if and only if every vniformly continuous pseudometric on it is bounded.

Proof. Atsuji showed [1] that a space is finitely chainable (uniformly bounded) if and only if every real-valued uniformly continuous function on it is bounded. Evidently an unbounded pseudometric would yield an unbounded real function, and conversely.

It was noted in [15] that a uniformly bounded space having a basis of star-finite uniform coverings is precompact, i.e., has a basis of finite coverings; more specifically, every star-finite uniform covering of a uniformly bounded space is finite. Hence a uniformly locally compact uniformly bounded space must be compact. We have further

5.2. An LCWU space having a basis of star-finite [star-bounded] uniform coverings has a basis which is a star-finite [star-bounded] collection.

and

5.3. A locally compact, uniformly contractible, complete space is compact.

Proof of 5.2. We need only a single star-finite [star-bounded] uniform covering $\left\{U_{\alpha}\right\}$ whose members admit an equiuniformly continuous family of contractions $h_{\alpha}$. Then the sets $h_{\alpha}\left(U_{\alpha} \times I\right) \supset U_{\alpha}$ are uniformly bounded; moreover, by equiuniform continuity, for each uniform covering $\mathscr{V}^{\prime}$ of the space there is $n$ such that any two elements of $\mathscr{Y}^{\prime}$ which have non-empty intersection with a common $U_{\alpha}$ can be joined by a chain of $n$ or fewer successively intersecting elements of $\mathscr{Y}$. Then take a basis consisting of coverings $\mathscr{V}^{-\lambda}$ each of which is finer than $\left\{U_{\alpha}\right\}$ and star-finite [star-bounded]. For each $\lambda$, each member of $\mathscr{V}^{\lambda}$ is contained in some $U_{\alpha}$ and meets no more other $U_{\beta}$ 's than $U_{\alpha}$ does. On the other hand, the members of $\mathscr{V}^{\lambda}$ which meet a given $U_{a}$ are all in the $n$th iterated star of some one member of $\mathscr{V}^{\lambda}$; thus they form a finite collection, and if $\mathscr{V}^{\lambda}$ is star-bounded then these collections also are of bounded size. 
Proof of 5.3. Let $X$ be complete and locally compact and let $h: X \times I \rightarrow X$ be a uniformly continuous contraction. Then $h(X \times\{1\})$ is compact, being a single point. Then cannot be a least $t$ in $[0,1]$ (different from 0$)$ such that $h(X \times\{t\})$ is precompact; for a precompact subset of a complete locally compact space has a compact uniform neighborhood. But it is also impossible that there is a greatest $t$ such that $h(X \times\{t\})$ is not precompact. For, if $S=h(X \times\{t\})$ is not precompact, there is a uniform covering of $X$ containing an infinite uniformly discrete subcollection $\left\{U_{i}\right\}$, each $U_{i}$ containing at least one point $p_{i}$ of $S$; if $\mathscr{V}$ is a star-refinement of this covering and $h(X \times\{t+\varepsilon\})$ is within $\mathscr{V}^{\text {r of }}$ $S$, then $h(X \times\{t+\varepsilon\})$ meets every $U_{i}$ and hence is not precompact. The only remaining possibility is that all $h(X \times\{t\})$, including $X$ itself, are precompact. Since $X$ is complete, it is compact.

In 5.2, evidently, local contractibility is not essential; only a suitable local boundness condition is needed. In 5.3, "uniformly contractible" cannot be replaced by "uniformly bounded". An example may be constructed as follows. Begin with the half-line $(1, \infty)$, with $d(x, y)=$ $\min (|x-y|, 1)$. At each $n=3,4 \cdots$, erect whiskers $W_{n i}$ for $i=1, \cdots, n-1$, of length $1-1 / i$ and reaching to within $1 / i$ of the point $i$. Let the distances not mentioned be the greatest compatible with the triangle inequality

Let us stress the following corollary to 5.3.

5.4. A locally compact ARU is compact. A locally compact ANRU is uniformly locally compact.

The first assertion follows at once from 5.3; the second can clearly be proved by a similar argument. 5.4 includeds the previous result that at least one extension space for collectionwise normal spaces (the real line [15]) cannot be an $A R U$ in any uniformity. There are also $A N R$ 's-indeed, neighborhood extension spaces for normal spaces-which cannot be $A N R U$ 's; for any open subset of a product of closed intervals is a neighborhood extension space for normal spaces, locally compact, but not paracompact if the product has uncountably many factors, and thus not uniformly locally compact in any uniformity. However, 5.4 was not needed for this; these spaces are not even complete in any uniformity.

5.5. Let $X$ be an $A N R U$ which is homeomorphic with a non-discrete separable metric space. Then the least cardinal number of a basis of uniform coverings of $X$ is a countable sum of smaller cardinals. Consequently if the power of the continuum is less than $\boldsymbol{\aleph}_{\omega}$, then $X$ is a metric uniform space.

Proof. Let $\mathscr{B}$ be a basis of uniform coverings of minimum cardinal 
$\boldsymbol{W}_{\alpha}$; this is an infinite cardinal since $X$ is not discrete. Well-order $\mathscr{B}$ into a sequence $\left\{\mathscr{U}^{\lambda} \mid \lambda<\omega_{\alpha}\right\}$. For each $\lambda$ select a uniformly continuous mapping $f_{\lambda}$ of $X$ onto a metric space $M_{\lambda}$ such that every subset of $X$ whose image under $f_{\lambda}$ has diameter $<1$ is a subset of some element of $\mathscr{\ell}^{\lambda}$. Moreover, let $f_{-1}$ be a uniformly continuous homeomorphism of $X$ onto a metric space $M_{-1}$; such a mapping exists, i.e., the given uniformity contains a metric uniformity, by [15, Lemma 3]. Then let $P_{\lambda}$ be the product of all the metric spaces $M_{\beta},-1 \leqq \beta \leqq \lambda$, and let $h_{\lambda}: X \rightarrow P_{\lambda}$ be the uniformly continuous homeomorphism whose coordinates are the mappings $f_{\beta}$. Let $X_{\lambda}=h_{\lambda}(X)$. Observe that $h_{\lambda}^{-1}: X_{\lambda} \rightarrow X$ is certainly not uniformly continuous, for $X_{\lambda}$ is a subset of a product of fewer than $\boldsymbol{\aleph}_{a}$ metric spaces and therefore has a basis of uniform coverings whose cardinal number is less than $\boldsymbol{\aleph}_{\alpha}$ (unless $\alpha=0$, in which case we have the result we wish to prove).

For $\lambda>\mu$ there is a uniformly continuous projection $p_{\lambda \mu}: X_{\lambda} \rightarrow X_{\mu}$, the restriction of the projection of $P_{\lambda}$ upon the partial product $P_{\mu}$; moreover, $p_{\lambda \mu}$ is a homeomorphism. We define a uniform space $Y$ whose points are the points of $X$ and of all $X_{\lambda},-1 \leqq \lambda<\omega_{\alpha}$, as follows. For any $\mu<\omega_{\alpha}$, for any uniform covering $\left\{U_{\beta}\right\}$ of $X_{\mu}$, for any choice of a uniform covering $\mathscr{V}_{\lambda}^{\wedge}$ of $X_{\lambda}$ for each $\lambda<\mu$, the following sets form a uniform covering of $Y$ :

(1) the members of the coverings $\mathscr{C}_{\lambda}, \lambda<\mu$;

(2) the sets $W_{\beta}$ consisting of $U_{\beta} \subset X_{\mu}, h_{\mu}^{-1}\left(U_{\beta}\right) \subset X$, and all $p_{\mu \mu}^{-1}\left(U_{\beta}\right)$, $\lambda>\mu$. The coverings just described clearly form a basis for a uniformity; by definition, this is the uniformity of $Y$.

The uniformity induced on $X$ as a subspace of $Y$ coincides with the given uniformity, since $\mathscr{B}$ is a basis. Since $X$ is an $A N R U$, there is a uniformly continuous retraction $r: U \rightarrow X$, where $U$ is some uniform neighborhood of $X$ in $Y$. But $U$ contains all $X_{\lambda}$ with indices $\lambda$ exceeding some fixed $\lambda_{0}$. For each point $p$ in $X$, consider the subspace $T_{p}$ of $U$ consisting of $p$ and the points $h_{\lambda}(p), \lambda>\lambda_{0}$. In $T_{p}$, every neighborhood of $p$ contains all $h_{\lambda}(p)$ for $\lambda$ exceeding some fixed $\lambda_{1}$. Now $r$ maps $T_{p}$ continuously into the metric space $X$, with $r(p)=p$. Unless $\boldsymbol{\aleph}_{\alpha}$ is a countable sum of smaller cardinals, $r$ is finally constant on $T_{p}$. Apply this to the points $p_{i}$ of some countable dense set; we find that for some $\lambda^{*}<\omega_{\alpha}$, for all $\lambda>\lambda^{*}, r\left(h_{\lambda}\left(p_{i}\right)\right)=p_{i}$ for all $i$. But $r$ is continuous, so that $r\left(h_{\lambda}(p)\right)=p$ for all $p$ in $X$, for any $\lambda>\lambda^{*}$. This contradicts uniform continuity of $r$; thus $\aleph_{a}$ must be a countable sum of smaller cardinals.

The last assertion follows from the remark that $\aleph_{\alpha}$ cannot exceed the power of the continuum; for any uniformity on the topological space $X$ is induced by a family of continuous mappings of $X$ into a universal separable metric space (say $l_{\infty}$ ), and there are only continuum-many such mappings. 
We note in conclusion that a discrete topological space obviously is not an $A N R U$ in any uniformity different from the discrete one, which is a metric uniformity.

\section{Dimension 1. This section is devoted to the proofs of}

6.1. THEOREM. Assuming the power of the continuum is less than $\aleph_{\omega}$, an ANRU homeomorphic with the real line must be uniformly equivalent with the real line. More generally, an ANRU homeomorphic with a 1-dimensional atriodic uniform complex must be a uniform complex.

and

\subsection{THEOREM. A 1-dimensional compact ANR must be metric.}

Proof of 6.1. A space $X$ which is homeomorphic with the line is uniformly equivalent with the line provided $X$ is metric, complete, uniformly locally connected, and has a star-bounded basis [14]. If $X$ is an $A N R U$ it must be complete and uniformly locally connected. By 5.5, $X$ must be metric. In view of 5.2 it remains only to show that every uniform covering $\mathscr{U}$ of $X$ has a star-bounded uniform refinement. Let $\mathscr{V}$ be a uniform star-refinement of $\mathscr{U}$ consisting of connected sets, i.e., intervals. For each $x$ in $X$ let $W_{x}$ be the star of $x$ with respect to $y^{\prime}$. Use some homeomorphism to identify the points of $X$ with real numbers, and consider $W_{0}$. Since $X$ is connected, there exists a point $x_{1}>0$, not in $W_{0}$, such that $W_{x_{1}}$ meets $W_{0}$. That is, there exist intervals $I_{0}$ and $I_{1}$ in $\mathscr{Y}^{-}$with $0 \in I_{0}, x_{1} \in I_{1}$, and $I_{0} \cap I_{1}$ not empty. Then $W_{1}=\left(0, x_{1}\right) \cup W_{x_{1}}$ is a subset of the star of $I_{1}$ with respect to $\mathscr{V}^{-}$, and thus of some element of $\mathscr{U}$. In the same manner we obtain $W_{2} \subset\left(x_{1}, \infty\right)$, and so on, and on the other side $W_{-1} \subset(-\infty, 0)$, and so on, ending with a covering $\mathscr{W}$ of density 2 which is finer than $\mathscr{U}$ but coarser than $\mathscr{Y}$. In particular, $\mathscr{W}$ is star-bounded and uniform, as required.

The phrase "More generally" in the statement is justified by the fact that every uniform complex homeomorphic with the line is uniformly equivalent with the line; we omit the proof of this.

The components $K_{\alpha}$ of a 1-dimensional atriodic complex may be lines, half-lines, segments or circles. If the complex $X$ is an $A N R U$ in some given uniformity, then the subspaces $K_{\alpha}$ are uniform complexes in the induced uniformity; this follows from compactness for the segments and circles, from the preceding (with the hypothesis $2 \boldsymbol{S}^{\circ}<\boldsymbol{\aleph}_{\omega}$ ) for the lines, and from virtually the same proof for the half-lines. To show that $X$ as a whole is a uniform complex is a routine exercise depending on the fact that $X$ must be $L C W U$; we omit the details.

For 6.2 we shall need some lemmas. The idea of the proof is simple. 
The $A N R$ 's in question are locally trees; from several equivalent definitions we choose to define a tree as a locally arcwise connected compact space in which every two points are joined by a unique arc. The key lemma peculiar to trees $X$ is that if $X$ has a dense subset of cardinal $m$ than $X$ can be embedded in a product of $m$ closed intervals. For $m=\boldsymbol{W}_{1}$, the product space has a countable dense subset. If $X$ is a neighborhood retract of the product it too has a countable dense subsets; then by the lemma, $X$ must be metric.

6.3. A 1-dimensional compact $A N R$ can be covered by open sets whose closures are trees.

Proof. First, it suffices to show that such a space $X$ can be covered by open locally arcwise connected sets in which each two points are joined by a unique arc; for any closed arcwise connected subset of such a set is locally arcwise connected because of the uniqueness, and the covering can be shrunk because of compactness. Then, since an $A N R$ and all its open subsets are locally arcwise connected, it suffices to show that each point $p$ has a neighborhood $U$ in which any two points are joined by a unique arc. But each $p$ has an arcwise connected neighborhood $U$ which is contractible in $X$. If joining arcs in $U$ were not unique, $U$ would contain a circle $S$. The identity mapping of $S$ to $S$ could be extended continuously over the 1-dimensional space $X$; since $S$ is contractible in $X$, this is absurd.

6.4. In a tree, every point has a basis of neighborhoods whose boundaries are finite sets.

Proof. Let $p$ be a point of the tree $X$ and $U$ an arcwise connected neighborhood of $p$. Consider the compact boundary $B$ of $U$. For each point $b$ of $B$, there is a unique arc $A$ joining $b$ to $p$, and a suitable point $c$ interior to $A$ and $U$ separates $b$ from $p$. For each such $c$, the set of all points of $X$ separated from $p$ by $c$ is open, since it is a union of components of $X-c$. Then finitely many points $c_{1}, \cdots, c_{n}$ of $U$ suffice to separate $p$ from every point of $B$. The component of $p$ in the complement of $\left\{c_{1}, \cdots, c_{n}\right\}$ is then a neighborhood of $p$ contained in $U$ whose boundary is finite.

In the next lemma, a branch point is a point whose complement has three or more components, an end point a point whose complement is connected. The order of a point is the number of components of its complement. An open arc which contains no branch point, but both of whose ends are branch or end points, will be called a maximal free arc. Obviously the maximal free arcs are pairwise disjoint open sets. 
6.5. For a tree $X$ and an infinite cardinal $m$, the following conditions are equivalent.

(a) $X$ contains no family of more than $m$ disjoint arcs.

(b) $X$ has no more than $m$ branch points and no point of order greater than $m$.

(c) X has a dense subset of cardinal $m$.

Proof. Assume (a). Evidently there is no point of order $>m$, since every component of its complement must contain an arc. Also, any one are $A$ in $X$ contains no more than $m$ branch points; for we can associate to each branch point $x$ in $A$ a closed arc $I(x)$ whose intersection with $A$ is exactly $x$, and these arcs will be disjoint. Moreover, for the same reason, if $S$ is an arcwise connected subset of $X$ then the closure of $S$ contains no more than $m$ branch points not in $S$. Then let $J$ be the set of all ordinals of cardinality $m$ or less. Let $A_{0}$ be a closed arc in $X$. Inductively, for each ordinal $\alpha$ in $J$, let $S_{\alpha}$ be the closure of the union of the $\operatorname{arcs} A_{\beta}, \beta<\alpha$. If $S_{\alpha}$ is not all of $X$, let $A_{\alpha}$ be a closed arc having exactly one end point (of the arc) in common with $S_{\alpha}$. Inductively, each $S_{\alpha}$ contains no more than $m$ branch points. But the induction must end before $J$ is exhausted, for the open arcs obtained from all $A_{\alpha}$ by removing the ends form a pairwise disjoint family. Then (b) is proved.

To deduce (c) from (b), observe that if $X$ is not just an arc, then each maximal free arc has a branch point at least at one end; thus there are no more than $m$ maximal free arcs. Each has a countable dense subset, and these together with the branch points make a dense subset of $X$.

Finally suppose $D$ is a dense set of cardinal $m$. Let $S$ be the union of all closed arcs both of whose ends are in $D$. Then any arc containing more than one point of $S$ contains one of the $m$ constituent arcs of $S$, and no disjoint family can contain more than $m$ of these. But the complement of $S$ cannot contain an arc $A$. For if $p, q, r$, are three points of $A, q$ separating $p$ from $r$, the dense set $D$ contains a point $p^{\prime}$ not separated from $p$ by $q$ and a point $r^{\prime}$ not separated from $r$ by $q$; hence $q$ separates $p^{\prime}$ from $r^{\prime}$ and the arc joining $p^{\prime}$ and $r^{\prime}$ contains the point $q$ of $A \cap S$. This completes the proof of 6.5.

6.6. A tree $X$ having a dense subset of cardinal $m$ is homeomorphic with a subset of a product of $m$ closed intervals.

Proof. The key conclusion from 6.5 is that there is a subset $D$ of cardinal $m$ such that every arc in $X$ contains a point of $D$; for this we may take the branch points and countable dense subsets of the maximal free arcs. Now for a homeomorphism into a product of $m$ intervals it suffices to have a family of $m$ continuous functions $f_{\alpha}: X \rightarrow[0,1]$ which 
separate points and closed sets. For each point $p_{0}$ of $D$ and each finite subset $\left\{p_{1}, \cdots, p_{n}\right\}$ of $D-p_{0}$, there is a continuous function $f_{\alpha}: X \rightarrow[0,1]$ which is 1 at $p_{0}$ and vanishes outside the component of $p_{0}$ in $X-\left\{p_{1}, \cdots, p_{n}\right\}$; by 6.4 , these functions suffice.

6.7. Every closed connected subset of a tree $X$ is a retract of $X$.

To retract $X$ upon the closed connected subset $S$, choose $s_{0} \in S$ and define $r(x)$ for each $x$ in $X$ as the first point of $S$ on the unique arc from $x$ to $s_{0}$; continuity is obvious.

Proof of 6.2. Suppose $X$ is a compact $A N R$ of dimension 1 which is not metric. Then $X$ has a finite covering by trees, and not all of these can be metric. Moreover, if we take a covering $\left\{T_{1}, \cdots, T_{n}\right\}$ by trees which is a shrinking of a covering by open sets whose closures are trees, then each $T_{1}$ is a neighborhood retract of $X$ and thus an $A N R$. Hence we obtain a tree $T$ which is an $A N R$ and contains at least $\aleph_{1}$ disjoint arcs. Let $S$ be the intersection of the closed connected subsets of $T$ which contain all the members of a certain family of $\boldsymbol{\aleph}_{1}$ disjoint arcs. Then $S$ is a tree, a retract of $T$; and the least cardinal of a dense subset of $S$ is exactly $\boldsymbol{\aleph}_{1}$. But by $6.6, S$ is homeomorphic with subset of a product $P$ of $\boldsymbol{\aleph}_{1}$ closed intervals. It is well known [19; Problem 3N] that $P$ has a countable dense subset. Since $S$ is an $A N R$, it is a retract of an open subset of $P$, and $S$ itself has a countable dense subset. The contradiction establishes the theorem.

\section{SPECIAL TOPICS}

\section{Inverse limits.}

7.1. Every complete uniform space is an inverse limit of ANRU's.

Proof. Let $Y$ be complete, and consider $Y$ as embedded in a product $P$ of function spaces $F_{\alpha}=U\left(D_{\alpha}, I\right)$, where $D_{\alpha}$ is discrete. We need only show that $Y$ is the intersection of a directed set of $A N R U$ 's containing it in $P$; then these spaces with inclusion mappings form an inverse mapping system with inverse limit $Y$. Let us call a canonical neighborhood of a point $p \in P$ the open neighborhood determined by any $\varepsilon>0$ and any finite set of indices $\alpha_{1}, \cdots, \alpha_{n}$, consisting of all those $q$ whose $\alpha_{i}$ th coordinate projections for $i=1, \cdots, n$, are all at distance $<\varepsilon$ from the corresponding projections of $p$. The complement in $P$ of one canonical neighborhood determined by $p$, $\varepsilon$, and $\alpha_{1}, \cdots, \alpha_{n}$, is a uniformly continuous retract of the complement of the smaller canonical neighborhood determined by $p, \varepsilon / 2$, and the same indices; thus it is an $A N R U$. Further, by a finite averaging procedure, one sees at once that the complement 
of a finite union of canonical neighborhoods of various points is also an $A N R U$. But the complements of finite unions of canonical neighborhoods disjoint from $Y$ constitute a directed set whose intersection is $Y$, as required.

It was noted in [3] that a uniform space $Y$ cannot be inverse limit of uniform complexes unless every uniform covering of $Y$ has a finitedimensional uniform refinement; and it was shown there that this condition is necessary and sufficient in the case that $Y$ is complete metric. If $Y$ is also $n$-dimensional, the complexes can be $n$-dimensional too. On the other hand, Mardešic has shown [21] that there exist 1-dimensional compact Hausdorff spaces which are not inverse limits of 1-dimensional complexes. In view of this rather surprising result, it is worth pointing out that complete $n$-dimensional spaces do possess all properties which are possessed by $n$-dimensional complexes and preserved under inverse limits, for such a space is an inverse limit of inverse limits of $n$-dimensional complexes. Mardešic has proved this in the compact case [21, Corollary 1]. More generally, we have

7.2. Let $X$ be a complete uniform space and $\left\{\mathscr{Z}^{\alpha}\right\}$ a basis of uniform coverings of $X$, each finite-dimensional. Then

(i) $X$ is an inverse limit of finite-dimensional uniform complexes;

(ii) $X$ is an inverse limit of inverse limits of nerves of coverings $\mathscr{U}^{\alpha}$.

The proof requires two lemmas. For (i), a finite product of finitedimensional uniform complexes is a finite-dimensional uniform complex. The required triangulation may be gotten by constructing the non-simplicial product complex whose cells are products of simplexes, and taking the first barycentric subdivision. For (ii), Morita's lemma: If $Y$ is the completion of $X$, every uniform covering $\left\{U_{a}\right\}$ of $X$ is the trace on $X$ of a uniform covering $\left\{V_{\alpha}\right\}$ of $Y$ with isomorphic nerve, and with each $U_{\alpha}$ dense in $V_{\alpha}$. This is provided in [25].

Now the proof of (i) is precisely parallel to the proof for the compact case [7, p. 284]; so we omit it.

Proof of 7.2 (ii). Every normal sequence $\subseteq$ of coverings from $\left\{\mathscr{U}^{\alpha}\right\}$ determines a metric quotient space $M(\Im)$ of $X$, and a projection $p(\mathfrak{S}): X \rightarrow M(\mathfrak{S})$. Let $N(\mathfrak{S})$ denote the completion of $M(\mathfrak{S})$. By Morita's lemma, $N(\subseteq)$ has a basis of uniform coverings with nerves isomorphic with the nerves of the coverings in $\subseteq$. Then by [3, p. 21], each $N(\subseteq)$ is an inverse limit of these nerves. But obviously the complete space $X$ is the inverse limit of the spaces $N(\mathfrak{S})$ under the natural mappings.

Note that either conclusion of 7.2 implies the hypothesis; specifically, for (i), $X$ must be complete and have a basis of finite-dimensional coverings, while for (ii), $X$ must be complete and have a basis of coverings 
whose nerves are embeddable in the representing complexes.

A space $Z$ is said to dominate $Y$ if there are mappings $p: Y \rightarrow Z$ and $h: Z \rightarrow Y$ such that $p: Y \rightarrow Y$ is homotopic to the identity. If $\mathscr{U}$ is a covering of $Y$ such that each path in the homotopy just mentioned is contained in some element of $\mathscr{U}$, then $Z$ is said to $\mathscr{U}$-dominate $Y$. In analogy with Hanner's theorem for separable metric $A N R$ 's [12; Theorem 6.1], we have

7.3. An ANRU in which every uniform covering has a finite-dimensional uniform refinement is $\mathscr{U}$-dominated, for each uniform covering Ql, by some finite-dimensional uniform complex.

We omit the details. The required homotopy will exist if the composition $h p$ is sufficiently near the identity mapping. If $p$ is a canonical mapping into the nerve of a covering, a suitable mapping $h$ can be obtained by applying Hahn's lemma to a discontinuous function taking each point $p$ of the nerve to some point in an element of the covering corresponding to a vertex of the carrier of $p$.

In separable metric (topological) spaces the converse is true [11]; it seems to be unknown whether the same can be said for finite-dimensional metric spaces. In general, the results of the last ten years on nonseparable metric spaces seem to depend rather heavily on the exisence of sufficiently many locally finite coverings and canonical mappings into their nerves. This we have at least in the uniform spaces which have bases of finite-dimensional uniform coverings; but of course we do not have the possibility of constructing a mapping by purely local arguments, and it appears that much more technique must be developed before many questions can be satisfactorily treated.

I should like to repeat the remark from [3] and [15] that the uniform complexes are clearly not the right concept for the infinite-dimensional case. The finite-dimensionality in 7.2 and 7.3 may very likely appear for no better reason than that we do not have the right uniformity for the complexes.

Looking beyond simplicial complexes, it is worth noticing that a space $Y$ which is $\mathscr{U}$-dominated (for every uniform covering $\mathscr{U}$ ) by a space satisfying Hahn's lemma will also satisfy Hahn's lemma. The homotopy is quite irrelevant for this; all that is needed is the possibility of approximating the identity mapping $Y \rightarrow Y$ by mappings $Y \rightarrow Z \rightarrow Y$, where $Z$ is a well-behaved space.

7.4. If $X$ is an inverse limit of spaces $X_{\alpha}$, with the projections $X \rightarrow X_{\alpha}$ onto, and $Y$ satisfies Hahn's lemma, then the mappings from $X$ to $Y$ which can be expressed as projection upon some $X_{a}$ followed by a mapping from $X_{a}$ to $Y$ form a dense subset of $U(X, Y)$.

Proof. For any mapping $f: X \rightarrow Y$ and any uniform covering $\mathscr{U}$ 
of $Y$ let $\mathscr{C}^{\prime}$ be a uniform star-refinement of $\mathscr{U}$ and $\mathscr{V}$ a uniform covering of $Y$ such that any function into $Y$ under which the inverse image of $\mathscr{Y}^{\prime}$ is a uniform covering is within $\mathscr{U}^{\prime}$ of a uniformly continuous function. Then $f^{-1}\left(\mathscr{V}^{n}\right)$ is a uniform covering of $X$; thus for some projection $\pi_{\alpha}: X \rightarrow X_{\alpha}$ and some uniform covering $\mathscr{W}$ of $X_{\alpha}, \pi_{\alpha}^{-1}(\mathscr{W})$ refines $f^{-1}\left(\mathscr{V}^{\prime}\right)$. Define a function $h: X_{\alpha} \rightarrow Y$ as follows: for each $p \in X_{\alpha}$ select $x \in X$ such that $\pi_{\alpha}(x)=p$, and let $h(p)=f(x)$. Each element $W$ of $\mathscr{W}$ then goes into $f\left(\pi_{\alpha}^{-1}(W)\right)$, which is a subset of an element of $\mathscr{V}^{2}$; thus there is a mapping $g: X_{\alpha} \rightarrow Y$ within $\mathscr{U}^{\prime}$ of $h$. Finally, $h \pi_{\alpha}$ is within $\mathscr{V}^{\prime \prime}$ of $f$ and $g \pi_{\alpha}$ is within $\mathscr{U}^{\prime}$ of $h \pi_{\alpha}$; thus $g \pi_{\alpha}$ is within $\mathscr{C}$ of the arbitrary mapping $f$, as required.

We say that a space $Y$ is an extension space for a class of spaces $X_{\alpha}$ provided every mapping of a subspace of any $X_{\alpha}$ into $Y$ can be extended to a mapping of $X_{\alpha}$ into $Y$.

7.5. If $Y$ is an ANRU and an extension space for certain spaces $X_{\alpha}$, then $Y$ is an extension space for all inverse limits of the spaces $X_{\alpha}$.

Proof. Observe that if $X$ is the limit space of the inverse mapping system $\left\{X_{\alpha} ; f_{\alpha \beta}\right\}$ then any closed subset $S$ of $X$ is the limit of $\left\{\pi_{\alpha}(S) ; f_{\alpha \beta}\right\}$. Any mapping $f: A \rightarrow Y, A \subset X$, can be extended at least over the closure $S$ of $A$, since $Y$ is complete. Then $f: S \rightarrow Y$ can by 7.4 be approximated arbitrarily closely by a mapping $g \pi_{\alpha}$, for some $g: \pi_{\alpha}(S) \rightarrow Y$. If the approximation is close enough, $g \pi_{\alpha}$ is homotopic to $f$, since $Y$ is $L C U$. But $g$ can be extended over $X_{\alpha}$, which yields an extension of $g \pi_{\alpha}$ over $X$; by the homotopy extension lemma, $f$ can be extended over $X$.

REMARKs. (1) 7.4 and 7.5 are done for real-valued functions in [3].

(2) Smirnov has shown [27] that a space $X$ has $\delta$-dimension at most $n$ if and only if the sphere $S^{n}$ is an extension space for $X$. Thus 7.5 yields as a corollary the lower semi-continuity of $\delta$-dimension on inverse limits. However, it is easy to prove this directly.

\section{Extensible continuous functions.}

8.1. Among continuous functions $f: A \rightarrow B$ in uniformisable spaces, the following conditions are equivalent.

(a) Whenever $A$ is a subspace of $X, B$ can be embedded in some $Y$ so that $f$ has a continuous extension over $X$ with values in $Y$.

(b) If $A$ is a closed subspace of $X$ then $B$ can be embedded in some $Y$ so that $f$ has a continuous extension over $X$ with values in $Y$.

(c) If $A$ is a closed subspace of $X$ then $B$ can be embedded in some $Y$, as a closed subspace, so that $f$ has a continuous extension over $X$ 
with values in $Y$.

(d) For any uniformity on $A$ there is a uniformity on $B$ making $f$ uniformly continuous.

(e) For every compactification $X$ of $A$ there is a compactification $Y$ of $B$ such that $f$ has a continuous extension over $X$ with values in $Y$.

Proof. The relations (a) $\Rightarrow(b) \Leftarrow \Rightarrow$ (c) are trivial. (For (b) $\Rightarrow$ (c), use the uniformisable quotient space of $X$ obtained by collapsing $A$ to a point.)

Next assume (b), and let $\mu$ be a uniformity on $A$. Let $\nu$ be a precompact uniformity coarser than $\mu$; let $C$ be the completion of $\nu A$; and let $W$ be the space of all ordinals less than or equal to some regular ordinal $\alpha$ larger than the cardinal of $A$. Let $X$ be the uniformisable space obtained from $C \times W$ by removing the points $(c, \alpha), c$ not in $A$. Then the points $(\alpha, \alpha)$ form a closed subspace of $X$ homeomorphic with $A$. In view of (b), there exist a space $Y$ containing $B$ and a continuous mapping $g: X \rightarrow Y$ extending $f$. Then $g$ is uniformly continuous with respect to the finest uniformities compatible with the topologies of $X$ and of $Y$; hense so is $f$. It remains to verify that the induced uniformity on $A$ is $\nu$, which is coarser than $\mu$. For this it sufficies to show that every continuous pseudometric $d$ on $X$, restricted to $A$, is $\nu$ - uniformly continuous. Now for any two points $(\alpha, \alpha),\left(a^{\prime}, \alpha\right)$, of $A \subset X$, consider the real-valued function $h$ on $W$ defined by $\left.h(\beta)=d(\alpha, \beta),\left(a^{\prime}, \beta\right)\right)$. It is continuous, hence finally constant; then there is an ordinal $\lambda<\alpha$ such that for $\lambda<\xi<h(\xi)=d\left((\alpha, \alpha),\left(a^{\prime}, \alpha\right)\right)$. If we select such a $\lambda$ for each pair $\left(a, a^{\prime}\right)$, we have a bounded set of ordinals less than $\alpha$; then there is $\lambda_{0}<\alpha$ such that $d\left(\left(\alpha, \lambda_{0}\right),\left(\alpha^{\prime}, \lambda_{0}\right)\right) \equiv d\left((\alpha, \alpha),\left(a^{\prime}, \alpha\right)\right)$. In particular, the restriction of $d$ to $A$ is continuously extensible over $C$ and therefore uniformly continuous on $\nu A$.

Next, (d) implies (e), by considering induced uniformities. Finally (e) implies (a). If $A \subset X$, let $Z$ be any compactification of $X$ and $C$ the closure of $A$ in $Z$. From (e), there is a compactification $K$ of $B$ such that $f$ can be extended to a continuous mapping $g: C \rightarrow K$. Let $Y$ be a cube containing $K$. Since $C$ is closed in $Z, g$ can be extended to $h: Z \rightarrow Y$, and $h \mid X$ is the required extension of $f$.

We shall call $f: A \rightarrow B$ intrinsically extensible if it satisfies the equivalent conditions of 8.1 .

The intrinsically extensible mappings fall into two types. We shall call $f: A \rightarrow B$ convergent at infinity, to a value $b$ in $B$, if for every neighborhood $U$ of $b$ there is a compact subet $K$ of $A$ such that $f(A-K) \subset$ $U$. We call $f$ proper if $f$ is closed (i.e., images of closed sets are closed) and inverse images of points are compact. 
The standard definition of proper mapping assumes both spaces are locally compact and requires that inverse images of compact sets are compact. This is weaker than the present definition if local compactness is not assumed, but equivalent if either $A$ or $B$ is locally compact or if $B$ is first countable [13]. From [13] we recall the connection between proper mappings $f: A \rightarrow B$ and the Cech extensions $f_{\beta}: \beta A \rightarrow \beta B$ over the Stone-Cech compactifications: $f$ is proper if and only if $f_{\beta}(\beta A-A) \subset$ $\beta B-B$. This is proved in [13] for onto mappings. To complete the proof we observe that $f: A \rightarrow B$ is proper if and only if $f: A \rightarrow f(A)$ is proper and $f(A)$ is closed in $B$. By [13, Lemma 1.5] $f$ is proper relative to $f(A)$ if and only if $f_{\beta}(\beta A-A)$ is disjoint from $f(A)$, and assuming this, one verifies at once that $f(A)$ is closed in $B$ if and only if $f_{\beta}(\beta A-A)$ is disjoint from $B$ as well.

It is evident, further, that $f: A \rightarrow B$ is convergent at infinity to $b$ if and only if $f_{\beta}(x)=b$ for all $x$ in $\beta A-A$.

8.2. THEOREM. Every function convergent at infinity is intrinsically extensible. An intrinsically extensible function which is not convergent at infinity is proper. Proper mappings with locally compact domain or range are intrinsically extensible.

Proof. If $f: A \rightarrow B$ is convergent at infinity to $b$, and $X$ is a compactification of $A$, we obtain a continuous extension of $f$ over $X$ (into any compactification of $B$ ) by defining $g(x)=f(x)$ for $x$ in $A, b$ for $x$ not in $A$.

Next suppose $f$ is neither convergent at infinity nor proper. Then $f_{\beta}$ takes some point $x_{1}$ of $\beta A-A$ to $b$ in $B$, and takes some other $x_{2}$ in $\beta A-A$ to $y \neq b$ in $\beta B$. There is a neighborhood $U$ of $b$ in $B$ whose closure in $\beta B$ does not contain $y$, and $f_{\beta}$ maps a neighborhood $V$ of $X_{2}$ into the complement of $U$; thus $f(V \cap A) \subset B-U$. Let $X$ be the quotient space of $\beta A$ obtained by identifying $x_{1}$ and $x_{2}$. A continuous extension of $f$ over $X$, into any compactification of $B$, would have to take the point $\left\{x_{1}, x_{2}\right\}$ to $f_{\beta}\left(x_{1}\right)$, which is $b$. However, every neighborhood of $\left\{x_{1}, x_{2}\right\}$ meets $V \cap A$ and therefore its image meets $B-U$. Thus $f$ cannot be extended continuously over $X$ and is not intrinsically extensible.

Next we show that if $f: A \rightarrow B$ is proper and $B$ is locally compact, $A$ is also locally compact. As remarked above, $f(A)$ is closed in $B$; thus $f(A)$ is locally compact. By $[13,2.2]$, so is $A$. On the other hand, assuming $A$ is locally compact, we conclude $[13,2.2]$ that $f(A)$ is locally compact. From either hypothesis we now have the conclusion that the set $C$ in $\beta B$ which is the closure of $f(A)$, less $f(A)$, is compact. Let $Y$ be the compactification of $B$ obtained from $\beta B$ by collapsing $C$ to a point. For any compactification $X$ of $A$, define $g: X \rightarrow Y$ by $g(x)=f(x)$ for $x$ 
in $A, g(x)=C$ for $x$ not in $A$. Since $A$ is open in $X, g$ is continuous at points of $A$. Since every neighborhood of $C$ contains all of $f(A)$ except a compact set, whose inverse image is compact, $g$ is continuous at points not in $A$. This completes the proof.

In view of 8.2 it is natural to inquire about extension in the category $\mathscr{L}$ of locally compact spaces and proper mappings. Defining an $A R$ of $\mathscr{L}$ as a space of $\mathscr{L}$ which is a retract by a mapping of $\mathscr{L}$ whenever it is embedded as a closed subspace of another space of $\mathscr{L}$, and similarly for other analogous terms, we have

8.3. Relative to $\mathscr{L}$, every mapping is intrinsically extensible. (That is, if $A, B, X$ are locally compact, $A$ closed in $X, f: A \rightarrow B$ proper, then $B$ can be embedded as a closed subspace of a locally compact space $Y$ and $f$ extended to a proper mapping $g: X \rightarrow Y$.) Hence every $A N R$ of $\mathscr{L}$ is a neighborhood extension space of $\mathscr{L}$. $\mathscr{L}$ has no $A R$ 's. It does have ANR's, including at least all locally compact $\sigma$-compact spaces which are ANR's of the category of locally compact spaces and continuous functions.

REMARKS. In the last assertion of 8.3, " $\sigma$-compact" is not necessary; I do not know whether it can be simply deleted. One can readily construct a special proof for at least the space obtained by deleting one point from any product of closed intervals. One can also replace " $\sigma$ compact" by "paracompact", but this is not actually a different result, for it follows easily from arguments in [23] that a paracompact $A N R$ in locally compact spaces (and continuous functions) must be $\sigma$-compact.

Proof of 8.3. Given three locally compact spaces, $A$ a closed subspace of $X$ mapped into $B$ by a proper mapping $f$, consider $\beta X$ and the closure $A^{-}$of $A$ in $\beta X$. From 8.2 , there is a compactification $B^{-}$of $B$ permitting a continuous extension $g: A^{-} \rightarrow B^{-}$of $f$. The sets $g^{-1}(p), p$ ranging over $g\left(A^{-}\right)$, form an upper semi-continuous decomposition of $A^{-}$. Therefore these sets and the single points of $\beta X-A^{-}$form an upper semi-continuous decomposition of $\beta X$, with quotient space $Z$ containing $g\left(A^{-}\right)$as a closed subset. Let $Z^{\prime}$ be the compact space obtained from the sum of $Z$ and $B^{-}$by identifying corresponding points of $g\left(A^{-}\right)$. The natural mapping $h: \beta X \rightarrow Z^{\prime}$ takes $A^{-}-A$ into $B^{-}-B$, since $f$ is proper; and $h$ takes the rest of $\beta X-X$ into $Z^{\prime}-B^{-}$. Further, $h(X)$ is disjoint from both $B^{-}-B$ and $h(\beta X-X)$. These last two sets are compact, since $B$ and $X$ are locally compact. Then let $Y=Z^{\prime}-h(\beta X-X)-$ $\left(B^{-}-B\right)$. $\quad Y$ is locally compact space containing $B$ as a closed subspace, and $h \mid X$ is a proper mapping into $Y$. This proves the first assertion, and the second follows trivially.

To show that $\mathscr{L}$ has no $A R$ 's it suffices to show that every space. 
$B$ can be embedded in a space $Y$ which has no proper mapping onto $B$. For this it suffices to take the sum of $B$ and a discrete space of larger power, since the inverse image of a point of $B$ must be compact and thus can contain only finitely many points of the discrete space.

Finally suppose $B$ is locally compact and $\sigma$-compact, and whenever $B$ is embedded as a closed subspace of a locally compact space $Y$ there is a continuous retraction $r$ of some neighborhood $U$ of $B$ onto $B$. We shall show that $U$ contains a neighborhood $V$ of $B$ such that $r \mid V$ is proper. Recall that $B$, being a Lindelof space, has the star-finite covering property [24], so that there is a star-finite covering $\left\{R_{\alpha}\right\}$ of $B$ consisting of open sets with compact closures. The star $S_{\alpha}$ of each $R_{\alpha}$ also has compact closure, by finiteness. The set $r^{-1}\left(S_{\alpha}\right)$ is a neighborhood of the closure of $R_{\alpha}$, and contains a compact neighborhood $V_{\alpha}$. Let $V$ be the union of all $V_{\alpha}$. To show that $r \mid V$ is proper, it suffices (since the spaces are locally compact) to show that the invere image of a compact subset $K$ of $B$ is compact. Since $K$ can meet only finitely many $R_{\alpha}$ 's, it suffices to show that $r^{-1}\left(R_{\alpha}^{-}\right) \cap V$ is compact. But it is closed, and it is contained in a finite union of compact sets, namely those $V_{\beta}$ for which $R_{\beta}$ is in the second iterated star of $R_{\alpha}$. This completes the proof.

Among the intrinsically extensible mappings, the constants of course permit mapping any space to any other. We note that the non-constant mappings convergent at infinity are closely related to the proper mappings, in the sense of

8.4. If $f: A \rightarrow B$ is convergent at infinity to $b$, then $f \mid A-f^{-1}(b)$ is proper into $B-b$, and $A-f^{-1}(b)$ is locally compact. The image $f(A)$ has compact closure.

Proof. A closed subset of the space $A-f^{-1}(b)$ is mapped into a subset of $B$ which contains all its limit points except perhaps $b$; for if $c$ is a point of $B-b$, there is a neighborhood $U$ of $b$ which does not have $c$ in its closure, and $f^{-1}(U)$ contains all of $A$ except a compact set. This show also that the inverse image of a point $c \neq b$ is compact. Moreover, for $x$ in $A$, if $f(x) \neq b$, there is a neighborhood of $f(x)$ whose inverse image is a neighborhood of $x$ contained in a compact set. Finally, $f(A)^{-}=f_{\beta}(\beta A)$.

Among the corollaries of 8.4 , observe that a nowhere locally compact space (such as the rational numbers) has no non-constant intrinsically extensible mapping into a locally compact space. On the other hand, whenever there is a proper mapping $g$ of a locally compact open subset $U$ of $A$ into a subset $C-b$ of $B$, where $C$ is compact and $b$ is a point of $C$, it is clear that extending $g$ over $A$ with $g(A-U)=b$ yields a mapping convergent at infinity to $b$. 
This amounts to a complete description of the intrinsically extensible mappings except for the additional conditions which must be imposed on proper mappings in the non-locally compact case. We conclude with a partial result on those conditions.

Let us call a proper mapping $f: A \rightarrow B$ one-to-one at infinity if the Čech extension $f_{\beta}$ maps $\beta A-A$ into $\beta B-B$ in a one-to-one manner (and hence, since $f_{\beta} \mid \beta A-A$ is also a proper mapping, homeomorphically). A mapping, of this sort is intrinsically extensible, as will be shown. However, we know that if $A$ is locally compact, no such condition is needed. Let $R(A)$ denote (as in [13]) the closed subset of $A$ consisting of all points at which $A$ is not locally compact. We say $f$ is one-to-one at infinity near $R(A)$ if the inverse images of points of $\beta B-B$ which are not single points all lie outside some neighborhood of $R(A)$ in $\beta A$, or in other words they lie in some compact subset of $\beta A-A$.

8.5. For a proper mapping $f: A \rightarrow B$ to be intrinsically extensible, it is sufficient that $f$ should be one-to-one at infinity near $R(A)$; if $A$ is first countable, the condition is also necessary.

Proof. First suppose $A$ is first countable and $f: A \rightarrow B$ is proper but not one-to-one at infinity near $R(A)$; thus for some $x \in R(A)$, every neighborhood of $x$ in $\beta A$ contains points of $\beta A-A$ which are not complete inverse images of their images. If $\left\{V_{i}\right\}$ is a countable basis at $x$ in $A$ then the sets $U_{i}=\left(V_{i}^{-}\right)^{0}$ form a basis at $x$ in $\beta A$. Choose points $x_{i}$ in $U_{i}-A$ and $y_{i} \neq x_{i}$ such that $f_{\beta}\left(y_{i}\right)=f_{\beta}\left(x_{i}\right)$. The points $y_{i}$ are in $\beta A-A$, since $f$ is proper, and since all their limit points $x^{\prime}$ satisfy $f_{\beta}\left(x^{\prime}\right)=f(x)$, they are in $A$. Then by choosing a subsequence we may assume the $y_{i}$ 's converge to a limit $x^{\prime}$, possibly $x$ itself. Evidently we may also assume all the points $x_{i}, y_{j}$, are distinct from each other. Then form a compactification $X$ of $A$ by the upper semi-continuous decomposition of $\beta A$ whose non-degenerate elements are certain two-point sets, specifically the pairs $\left\{x_{2 n}, x_{2 n+1}\right\}$ and the pairs $\left\{y_{2 n-1}, y_{2 n}\right\}$. Since the $x_{i}$ and the $y_{j}$ form two convergent sequences, the decomposition is upper semi-continuous and yields a compactification $X$. If $f$ could be extended to a mapping $g$ of $X$ into some compactification $Y$ of $B$, we should have $g\left(\left\{y_{1}, y_{2}\right\}\right)=g\left(\left\{x_{2}, x_{3}\right\}\right)$ since $f_{\beta}\left(x_{2}\right)=f_{\beta}\left(y_{2}\right), g\left(\left\{x_{2}, x_{3}\right\}\right)=g\left(\left\{y_{3}, y_{4}\right\}\right)$ since $f_{\beta}\left(x_{3}\right)=$ $f_{\beta}\left(y_{3}\right)$, and so on. Then $g$ would be constant on all these points, the value would be $g(x)=f(x) \in B$, and since $\left\{y_{1}, y_{2}\right\}$ is the limit of a net of points $p_{\alpha}$ of $A$ such that $f\left(p_{\alpha}\right)$ does not converge to $f(x)$, this is a. contradiction.

The other half of the proof depends on

8.6. If $G$ and $H$ are two upper semi-continuous decompositions of a normal space and every non-degenerate element of $G$ is disjoint from 
every non-degenerate element of $H$, then the decomposition whose nondegenerate elements are the non-degenerate elements of $G$ and of $H$ is upper semi-continuous.

The proof of this is a routine exercise, and we omit it.

Now suppose $f: A \rightarrow B$ is proper and one-to-one at infinity near $R(A)$, and let $X$ be any compactification of $A$. There is a compact subset $K$ of $\beta A-A$ which contains all the non-degenerate inverse images of points of $\beta B-B$ under $f_{\beta}$. Let $\pi$ be the natural mapping of $\beta A$ onto $X$, and $L=\pi^{-1}(\pi(K))$. Let $X_{0}$ be the quotient space of $\beta A$ by the decomposition whose only non-degenerate element is $L$, and $X_{1}$ the quotient space of $X$ by the decomposition whose only non-degenerate element is $\pi(K)=\pi(L)$. Then we have quotient mappings $\lambda: \beta A \rightarrow X_{0}, \alpha: X \rightarrow X_{1}$, and $g: X_{0} \rightarrow X_{1}$, the latter associated with an upper semi-continuous decomposition $G$ of $X_{0}$ whose non-degenerate elements are all contained in $X_{0}-\lambda(A)$. We obtain another upper semi-continuous decomposition $H$ of $X_{0}$, with all its non-degenerate elements contained in $\lambda(A)$, as follows. Let $Y_{0}=f_{\beta}(\beta A) \subset \beta B$. Let $Y_{1}$ be the quotient space of $Y_{0}$ by the decomposition whose only non-degenerate element is $f_{\beta}(L)$. Then the decomposition of $\beta A$ associated with $\beta A \rightarrow Y_{0} \rightarrow Y_{1}$ is coarser than the decomposition associated with $\lambda: \beta A \rightarrow X_{0}$, and therefore there is a quotient mapping $h: X_{0} \rightarrow Y_{1}$. All the non-degenerate inverse sets under $\beta A \rightarrow Y_{0} \rightarrow Y_{1}$ are contained in $A$, with the exception of $L$; therefore the decomposition $H$ associated with $h$ satisfies 8.6, and there is a quotient mapping $q: X_{0} \rightarrow Y_{2}$ whose non-degenerate sets $g^{-1}(p), p \in X_{1}-g \lambda(A)$, and the non-degenerate sets $\lambda f^{-1}(b), b \in B$. There is also a quotient mapping $Y_{1} \rightarrow Y_{2} ; \quad Y_{2}$ is the quotient space of $Y_{0}$ by an upper semicontinuous decomposition; and $Y_{0}$ is a closed subspace of $\beta B$. Therefore the decomposition of $\beta B$ whose non-degenerate elements are those subsets of $Y_{0}$ which are inverse sets under $Y_{0} \rightarrow Y_{1} \rightarrow Y_{2}$ is upper semicontinuous and yields a quotient space $Y$ of $\beta B$. Inspection of the definitions shows that the quotient mapping $\beta B \rightarrow Y$ embeds $B$ homeomorphically, so that $Y$ is compactification of $B$. Again, $Y_{2} \subset Y$ is the quotient of $X_{0}$ by a coarser decomposition than $G$, and therefore there is a quotient mapping $j: X_{1} \rightarrow Y_{2} \subset Y$. Then $j \alpha$ maps $X$ continuously into $Y$, and $j \alpha \mid \pi(A)$ agrees with $f$, as required.

9. Adequacy. If $B$ is a collection of objects of a category $\mathscr{C}$, and $X$ and $Y$ are two objects of $\mathscr{C}$, a right transformation from $X$ to $Y$ over $B$ is defined [17] as a function $\alpha$ which takes each mapping $f$ in $\mathscr{C}$ whose domain $W$ is in $B$ and whose range is $X$ to a mapping $\alpha(f): W \rightarrow Y$, satisfying $\alpha(f e)=\alpha(f) e$ for every $e$ in $\mathscr{C}$ which has range $W$ and domain an element of $B$. This rather formidable definition is really quite natural; any mapping $a: X \rightarrow Y$ defines a right transformation $\alpha$ by $\alpha(f)=a f$, and the question whether the converse is 
true is a precise form of the question whether there are enough mappings of "standard objects" (objects of $B$ ) into $X$ to determine $X$. In particular, if $X$ itself is in $B$, then each right transformation $\alpha$ from $X$ to any $Y$ over $B$ is defined on the identity $i: X \rightarrow X$ and is induced by the mapping $\alpha(i): X \rightarrow Y$. In general, $B$ is said to be left adequate in $\mathscr{C}$ if for every $X$ and $Y$, each right transformation from $X$ to $Y$ over $B$ is induced by a unique mapping in $\mathscr{C}$. In this paper we shall use also the localized notion: $B$ is left adequate for $X$ (with respect to $\mathscr{C}$ ) if the right tranformations from $X$ to each $Y$ over $B$ correspond in a one-to-one onto manner with mappings from $X$ to $Y$.

The notions of left transformation, right adequate in, and right adequate for are defined dually. We shall soon see (9.1) that in topology and in uniform spaces, it is only this half of the idea of adequacy that really presents new problems. To insure against confusion we describe a left transformation from $X$ to $Y$ over $B$ more fully; it takes each mapping $Y \rightarrow Z, Z$ in $B$, to a mapping $X \rightarrow Z$, and commutes with left multiplication by mappings $Z \rightarrow A$ in $B$.

We shall be considering the category of all uniform spaces with all uniformly continuous functions, the category of all uniformisable spaces with all continuous functions, and some full subcategories of these (i.e., subcategories which include all mappings between their own objects). For short, we shall specify the category in each case by specifying its objects, letting the mappings be understood.

9.1. Among uniformisable spaces or among uniform spaces, a class $B$ of objects is left adequate for an object $X$ if and only if $X$ is a quotient of the sum of a family of objects of $B . \quad B$ is right adequate for $X$ if and only if $X$ is a subspace of a product of spaces in $B$ and every left transformation from $a$ one-point space $P$ to $X$ over $B$ is induced by a mapping from $P$ to $X$. This implies that $X$ is a closed subspace of a product of spaces in $B$.

Proof. Suppose $X$ is a quotient of the sum $\Sigma$ of a family of objects $S_{\lambda}$ of $B$. For any right transformation $\alpha$ from $X$ to $Y$ over $B$, in topological spaces, it is shown in $[17,2.4]$ that there is a unique function $a$ from $X$ to $Y$, not necessarily continuous, such that $\alpha(f)=a f$ identically. This induces a mapping $\Sigma \rightarrow X \rightarrow Y$, which is in the category since $\Sigma$ is sum and all $\alpha(f)$ are in the category; since $X$ is a quotient, this implies $a$ is in the category.

Suppose $X$ is not a quotient of the sum of any family of objects in $B$. Consider each mapping $f$ of a space $W$ of $B$ into $X$. There is a quotient space $Q(f)$ of $W$ by the equivalence relation $f(p)=f(q)$; moreover, $Q(f)$ has the same cardinal number as $f(W)$, which is at most the cardinal of $X$. Then among the class of spaces $Q(f)$ we can find 
a set $\left\{Q_{\lambda}\right\}$ such that every $Q(f)$ is equivalent (topologically/uniformly) to some $Q_{\lambda}$. For each $Q_{\lambda}$ select some $f=f_{\lambda}$ such that $Q_{\lambda}=Q(f)$, and let $W_{\lambda}$ denote the domain of this $f_{\lambda}$. Then let $X^{\prime}$ be the quotient of the sum $S$ of all $W_{\lambda}$ by the equivalence relation $R$ defined as follows: for $p \in W_{\lambda} \subset S$ and $q \in W_{\mu} \subset S, p R q$ provided $f_{\lambda}(p)=f_{\mu}(q)$. Now $X^{\prime}$ is a quotient of sum of spaces of $B$, and thus is certainly not equivalent to $X$. However, all spaces of $B$ have "the same" mappings into $X^{\prime}$ as into $X$. More precisely, the right transformation $\alpha$ from $X^{\prime}$ to $X$ over $B$ induced by the natural mapping $a$ has an inverse $\beta$; if $B$ is left adequate for $X, \beta$ is induced by a mapping $b: X \rightarrow X^{\prime}$. Then $\alpha \beta$ is induced by $a b$; but $\alpha \beta$ is induced by the identity $i$ : $X \rightarrow X$, and hence $a b=i$. Similarly $b a$ is an identity, and $a$ is an equivalence; a contradiction.

If $X$ is not a closed subspace of a product of spaces in $B$, a similar argument applies. Any mapping $h: X \rightarrow T$ of $X$ into a product of spaces in $B$ maps $X$ onto a space $h(X)$ of cardinal number no greater than that of $X$; moreover, the closure of $h(X)$ in $T$ has limited size (at most $2^{2^{m}}$ if $m$ is the cardinal of $X$ ), so that there is no more than a set of essentially different possibilities and there is a mapping $k: X \rightarrow X^{\prime}$ upon a dense subset of a space $X^{\prime}$ which is a closed subspace of a product of spaces in $B$, inducing a left transformation from $X$ to $X^{\prime}$ over $B$ which has an inverse. Again, if $X$ is not equivalent to $X^{\prime}$, this inverse is a left transformation which cannot be induced by a mapping.

If $B$ is right adequate for $X$, then in particular every left transformation from a one-point space to $X$ over $B$ is induced by a mapping.

To conclude we need only show that if $X$ is a subspace of a product $T$ of spaces $Z_{\lambda}$ in $B$, and left transformations from a one-point space $P$ to $X$ over $B$ are induced by mappings, then each left transformation $\alpha$ from any space $W$ to $X$ over $B$ is induced by a unique mapping $a: W \rightarrow X$. Uniqueness is trivial, for the coordinate projections $\pi_{\lambda}: X \rightarrow Z_{\lambda}$ must satisfy $\pi_{\lambda} a=\alpha\left(\pi_{\lambda}\right)$, which completely determines $a$. On the other hand, a function $a$ on $W$ with values in the product space $T$ is defined by defining each coordinate $\pi_{\lambda} a$ to be $\alpha(\pi): W \rightarrow Z_{\lambda}$. To prove that $a(W)$ is contained in $X \subset T$, consider any point $p$ of $W$. There is a mapping $h: P \rightarrow W$ taking the value $p$. The left transformation $\beta$ from $P$ to $X$ defined by $\beta(g)=\alpha(g) h$ is by hypothesis induced by a mapping $b: P \rightarrow X$, i.e., $\alpha(g) h=g b$ for all $g: X \rightarrow Y, Y$ in $B$. In particular each $\pi_{\lambda} a h=$ $\alpha\left(\pi_{\lambda}\right) h=\pi_{\lambda} b$, so that every coordinate $\pi_{\lambda}(\alpha(p))$ is the same as the corresponding coordinate of $b(P)$, which is a point of $X$. Further, $a$ is a mapping in the category since all its coordinates are. It remains only to verify that for every $g: X \rightarrow Y, Y$ in $B, \alpha(g)$ is in fact the mapping $g a: W \rightarrow Y$; but this involves no more than $\alpha(g)(p)=g a(p)$ for each point $p$ of $W$, which is verified at once. This completes 9.1.

It is shown in [17] that a closed interval $I$ is not right adequate 
for a square $I^{2} ; I^{2}$, however, is right adequate for all compact spaces. In general, a sequence need not be right adequate even for its closed subspaces. DeGroot has given an example of a space $Y$ which has no continuous mappings into itself except the identity and the constant functions [10]; consequently $Y$ is not even right adequate for a twopoint space.

9.2. A uniform or uniformisable space $Y$ is right adequate for all its closed subspaces if either $Y$ contains an arc or $Y$ has covering dimension ( $\delta$-dimension) zero. The spaces in an inverse mapping system are right adequate for the inverse limit if either at least one of them contains an arc or all of them have covering dimension ( $\delta$-dimension) zero.

Proof. Let $S$ be a closed subspace of $Y$, with an embedding $e: S \rightarrow Y$. By 9.1 we need only consider left transformations $\alpha$ from a one-point space $P$ to $S$ over $Y$. The mapping $\alpha(e): P \rightarrow Y$ maps $P$ upon some point $p$ of $Y$. If $p$ were not in $S$, either hypothesis (arc or dimension zero) would imply the existence of a mapping $g: Y \rightarrow Y$ taking all of $S$ to one point $s$ and $p$ to another point $t$. Then $\alpha(g e)$ must map $P$ to $t$. But consider the constant function $h: Y \rightarrow Y$ with value $s$; $h g e=g e$, so that $\alpha(h g e)$ maps $P$ to $t$, unlike $h \alpha(g e)$. The contradiction shows that $p$ is in $S$. It remains to verify that for any $f: S \rightarrow Y$, the point range $r$ of $\alpha(f)$ is $f(p)$. As before, if $r$ and $f(p)$ differed there would be a mapping $g: Y \rightarrow Y$ with $g(r) \neq g f(p)$ but with $g(Y)$ contained in an arc or in a two-point space, the latter case arising when $Y$ is zero-dimensional, so that in either case every mapping of a closed subspace of $Y$ into $g(Y)$ can be extended. If $h: Y \rightarrow g(Y)$ is an extension of $g f$, we have $h e=g f$. Then $\alpha(h e)$ must be at once $h \alpha(e)$, mapping $P$ to $g f(p)$, and $g \alpha(f)$, mapping $P$ to $g(r)$. This proves the assertions about subspaces. The argument for inverse limits is similar, except that the determination of a point $p$ in the limit space by its coordinate projections $\alpha\left(\pi_{\lambda}\right)$ follows at once from the relations $\alpha\left(f_{\lambda \mu} \pi_{\lambda}\right)=f_{\lambda \mu} \alpha\left(\pi_{\lambda}\right)$.

From 9.1 and 9.2 we have at once

9.3. ANRU's (or $A R U$ 's) are right adequate for a uniform space $X$ if and only if $X$ is complete.

As for the arc $I$, the theorem is

9.4. An arc is right adequate for 1-dimensional compact spaces.

I do not know whether there are any spaces of dimension greater than 1 for which the arc is right adequate. The proof of 9.4 rests on the lemma 
9.4. a. An arc is right adequate for 1-dimensional finite complexes.

This is not difficult to prove, using a finite-to-one mapping of the complex upon $I$. We omit the details.

Now 9.4 would follow from 9.4. a. and 9.2, by Mardesic's theorem [21], if we had transitivity of adequacy. It is shown in [17] that relative adequacy ( $\mathscr{A}$ a right adequate subcategory of $\mathscr{B}$, and $\mathscr{B}$ of $\mathscr{C}$ ) is not transitive. It is also generally false that in $\mathscr{D}, \mathscr{A}$ being right adequate for each object of $\mathscr{B}$ and $\mathscr{B}$ for each object of $\mathscr{C}$ implies right adequacy of $\mathscr{A}$ for each object of $\mathscr{C}$. But we have

9.5. Right adequacy is transitive when it is monotonic, and this holds for zero-dimensional spaces, and for spaces containing arcs.

To be precise:

9.5. a. In any category $\mathscr{C}$, suppose that the class of objects $B$ is right adequate for the object $X$, and the class $A$ is right adequate for each object of $B$. Suppose further that every mapping from $X$ to an object of $A$ is factorable across an object of $B$. (This happens in particular when $B$ contains $A$.) Then $A$ is right adequate for $X$.

The proof is a straightforward computation which we omit. Note that since the category is arbitrary, the corresponding result for left adequacy follows by duality.

9.5. b. In the category of uniform or of uniformisable spaces, suppose that the class $A$ of spaces is left [right] adequate for $X$, and that $B$ is a class of spaces containing $A$. Then [if every space in $B$ is zero-dimensional, or if one in $A$ contains an arc] $B$ also is left [right] adequate for $X$.

Here the assertion about left adequacy is an immediate consequence 9.1. The result on right adequacy is deduced from 9.1 in the same way as 9.2. This completes the proof of 9.4.

Next, for the two dimensional spaces. It is clear that in uniform spaces the 2-dimensional ones cannot be right adequte, since the property of having a basis of finite-dimensional uniform coverings is preserved under the formation of products and subspaces. We shall show

9.6. 2-dimensional uniform complexes are right adequate for complete uniform spaces which have bases of finite-dimensional uniform coverings. In uniformisable spaces, 2-dimensional $C W$-complexes are right adequate for topologically complete spaces.

I do not know whether incomplete 2-dimensional spaces are right adequate even for incomplete 3 -dimensional spaces. To conclude, observe 
that 0-dimensional spaces are left adequate for all spaces; and one can easily work out further details on the left.

Proof of 9.6. For the first part, in view of 7.2, it suffices to show that 2-dimensional complexes are right adequate for finite-dimensional complexes $K$. Obviously $K$ can be embedded in a product of 2-dimensional complexes. It remains to consider left transformations $\alpha$ from a point $P$ to $K$. We shall associate with $\alpha$ a Cauchy filter, hence a point in $K$.

For the rest of the first part, it is not essential that $K$ is a uniform complex. What is essential is

(*) In a uniform space having a basis of finite-dimensional uniform coverings, a filter which includes an element of each 1-dimensional uniform covering is Cauchy.

For $(*)$, it suffices to show that a finite-dimensional uniform covering $\mathscr{U}$ has a refinement which is a finite intersection of 1-dimensional uniform coverings $\mathscr{C}_{i}$. It is proved in [14] that $\mathscr{U}$ has a uniform refinement which is a finite union of uniformly discrete collections $\mathscr{W}_{i}$. Evidently we may assume the union of the $\mathscr{W}_{i}$ is a strict shrinking of $\mathscr{C l}$, i.e., the stars of the elements of all $W_{i}$ with respect to some uniform covering $\mathscr{Z}$ still form a refinement of $\mathscr{U}$; and we may assume each collection $\mathscr{S}_{i}=\left\{\operatorname{St}(W, \mathscr{Z}): W \in \mathscr{W}_{i}\right\}$ is still disjoint. Then let $\mathscr{Y}_{i}$ consist of $\mathscr{S}_{i}$ and the complement of the union of $\mathscr{W}_{i}$.

Resuming with the left transformation $\alpha$, consider the family of all $M \subset K$ such that for each mapping $f$ of $K$ into a 1-dimensional complex, $\alpha(f)$ maps $P$ to a point of $f(M)$. The desired filter $\mathscr{F}$ will be the family of all uniform neighborhoods of such sets $M$. Indeed, trivial arguments show that $\mathscr{F}$ is a filter (since $\alpha$ is defined for all mappings of $K$ into 2-dimensional complexes) and that $\mathscr{F}$ includes an element of each 1-dimensional uniform covering. Then $\mathscr{F}$ converges to a unique point $p$ of $K$; and further routine arguments show that for each $f: K \rightarrow T, T$ a 2-complex, $f$ converges on $\mathscr{F}$ to $\alpha(f)$, i.e., $\alpha(f)=f(p)$.

For the second half of 9.6, we need only establish the analogue of (*): in any uniformisable space, a filter which includes an element of each 1-dimensional normal covering includes an element of every normal covering. Shirota has already done this with a possible exception for cardinal numbers which are measurable in the sense of Ulam (see [26]), using only countable 1-dimensional coverings. In particular, if the filter $\mathscr{F}$ includes an element of each 1-dimensional normal covering then it includes an element of each countable normal covering. But every normal covering has a refinement which is a countable union of collections $\mathscr{W}_{i}^{i}$ each of which is discrete in the finest uniformity on the space; this result is an easy consequence of A. H. Stone's work [28], but for a precise statement see [8, Theorem 4.4]. Then the sets $U_{i}$, the unions 
of the collections $\mathscr{H}_{i}$, form a countable normal covering; so $\mathscr{F}$ contains some $U_{i}$. Since $\mathscr{W}_{i}$ is discrete, there is a normal covering $\mathscr{Z}$ such that the stars of the elements of $\mathscr{W}_{i}$ with respect to $\mathscr{Z}$ are still discrete. Then these stars and the complement of $U_{i}$ form a 1-dimensional normal covering $\mathscr{Y}$. $\mathscr{F}$ contains some element of $\mathscr{Y}$, and $\mathscr{F}$ does not contain the complement of $U_{i}$; hence $\mathscr{F}$ contains some element of $\mathscr{W}_{i}$, as was to be shown.

9.7. COROLlary. Every completely uniformisable space is homeomorphic with an inverse limit of finite-dimensional uniform complexes.

Proof. By 7.2, we need only show that certain finite-dimensional coverings form a basis for a complete uniformity on the space; and this was done in the proof of 9.6 .

\section{REFERENCES}

1. M. Atsuji, Uniform continuity of continuous functions of metric spaces, Pacific J. Math. 8 (1959), 11-16.

2. K. Borsuk, Sur un espace localement contractible qui n'est pas un retracte absolu de voisinage, Fund. Math. 35 (1948), 175-580.

3. H. Corson and J. Isbell, Some properties of strong uniformities, Quart. J. Math. (2) 11 (1960), 17-33.

4. M. Day, Normed Linear Spaces, Berlin, 1958.

5. J. Dugundji, An extension of Tietze's theorem, Pacific J. Math. 1 (1951), 353-368.

6. - Absolute neighborhood retracts and local connectedness in arbitrary metric spaces, Comp. Math. 13 (1958), 229-246.

7. S. Eilenberg and N. Steenrod, Foundations of Algebraic Topology, Princeton, 1952.

8. S. Ginsburg and J. Isbell, Some operators on uniform spaces, Trans. Amer. Math. Soc. 93 (1959), 145-168.

9. A. Gleason, Projective topological spaces, Ill. J. Math. 2 (1958), 482-489.

10. J. DeGroot, Groups represented by homeomorphism groups, I, Math. Ann. 138 (1959), 80-102.

11. H. Hahn, Über halbstetige und unstetige Funktionen, Sitzungsb. Akad. Wien 126 (1917), 91-110.

12. O. Hanner, Some theorems on absolute neighborhood retracts, Ark. Mat. 1 (1951), 389408.

13. M. Henriksen and J. Isbell, Some properties of compactifications, Duke Math. J. 25 (1958), 83-105.

14. J. Isbell, Euclidean and weak uniformities, Pacific J. Math. 8 (1958), 67-86.

15. — On finite-dimensional uniform spaces, Pacific J. Math. 9 (1959), 107-121.

16. - Embeddings of inverse limits, Ann. of Math. (2) 70 (1959), 73-84.

17. - Adequate subcategories, Ill. J. Math. 4 (1960), 541-552.

18. M. Katětov, On real-valued functions in topological spaces, Fund. Math. 38 (1951), 8591.

19. J. Kelley, General Topology, New York, 1955.

20. C. Kuratowski, Quelques problèms concernant les espaces metriques non-separables, Fund. Math. 25 (1935), 534-545.

21. S. Mardešić, On covering dimension and inverse limits of compact spaces, Ill. J. Math. 4 (1960), 278-291. 
22. E. McShane, Extension of range of functions, Bull. Amer. Math. Soc. 40 (1934), 837842.

23. E. Michael, Some extension theorems for continuous function, Pacific J. Math. 3 (1953), 789-806.

24. K. Morita, Star-finite coverings and the star-finiteness property, Math. Japan. 1 (1948), 60-68.

25. - On the simple extension of a space with respect to a uniformity, I. Proc. Japan Acad. 27 (1951), 65-72.

26. T. Shirota, A class of topological spaces, Osaka Math. J. 4 (1952), 23-40.

27. Yu. Smirnov, On the dimension of proximity spaces, Mat. Sb. 38 (1956), 283-302.

28. A. Stone, Paracompactness and product spaces, Bull. Amer. Math. Soc. 54 (1948), 977982.

29. M. Stone, Applications of the theory of Boolean rings to general topology, Trans. Amer. Math. Soc. 41 (1937), 375-481.

UNIVERSITY OF WASHINGTON AND

PURDUE UNIVERSITY 


\title{
PACIFIC JOURNAL OF MATHEMATICS
}

\author{
EDITORS
}

\author{
Ralph S. Phillips \\ Stanford University \\ Stanford, California \\ F. H. Brownell \\ University of Washington \\ Seattle 5, Washington
}

\author{
A. L. Whiteman \\ University of Southern California \\ Los Angeles 7, California \\ L. J. PAige \\ University of California \\ Los Angeles 24, California
}

ASSOCIATE EDITORS
E. F. BECKENBACH
D. DERRY
H. L. ROYDEN
E. G. STRAUS
T. M. CHERRY
M. OHTSUKA
E. SPANIER
F. WOLF

\section{SUPPORTING INSTITUTIONS}

\author{
UNIVERSITY OF BRITISH COLUMBIA \\ CALIFORNIA INSTITUTE OF TECHNOLOGY \\ UNIVERSITY OF CALIFORNIA \\ MONTANA STATE UNIVERSITY \\ UNIVERSITY OF NEVADA \\ NEW MEXICO STATE UNIVERSITY \\ OREGON STATE COLLEGE \\ UNIVERSITY OF OREGON \\ OSAKA UNIVERSITY \\ UNIVERSITY OF SOUTHERN CALIFORNIA
}

\author{
STANFORD UNIVERSITY \\ UNIVERSITY OF TOKYO \\ UNIVERSITY OF UTAH \\ WASHINGTON STATE COLLEGE \\ UNIVERSITY OF WASHINGTON \\ * * * * \\ AMERICAN MATHEMATICAL SOCIETY \\ CALIFORNIA RESEARCH CORPORATION \\ HUGHES AIRCRAFT COMPANY \\ SPACE TECHNOLOGY LABORATORIES \\ NAVAL ORDNANCE TEST STATION
}

Mathematical papers intended for publication in the Pacific Journal of Mathematics should be typewritten (double spaced), and the author should keep a complete copy. Manuscripts may be sent to any one of the four editors. All other communications to the editors should be addressed to the managing editor, L. J. Paige at the University of California, Los Angeles 24, California.

50 reprints per author of each article are furnished free of charge; additional copies may be obtained at cost in multiples of 50 .

The Pacific Journal of Mathematics is published quarterly, in March, June, September, and December. The price per volume (4 numbers) is $\$ 12.00$; single issues, $\$ 3.50$. Back numbers are available. Special price to individual faculty members of supporting institutions and to individual members of the American Mathematical Society: $\$ 4.00$ per volume; single issues, $\$ 1.25$.

Subscriptions, orders for back numbers, and changes of address should be sent to Pacific Journal of Mathematics, 103 Highland Boulevard, Berkeley 8, California.

Printed at Kokusai Bunken Insatsusha (International Academic Printing Co., Ltd.), No. 6, 2-chome, Fujimi-cho, Chiyoda-ku, Tokyo, Japan.

\section{PUBLISHED BY PACIFIC JOURNAL OF MATHEMATICS, A NON-PROFIT CORPORATION}

The Supporting Institutions listed above contribute to the cost of publication of this Journal, but they are not owners or publishers and have no responsibility for its content or policies. 


\section{Pacific Journal of Mathematics}

\section{Vol. 11, No. 2 December, 1961}

Tsuyoshi Andô, Convergent sequences of finitely additive measures........

Richard Arens, The analytic-functional calculus in commutative topological algebras..........................................

Michel L. Balinski, On the graph structure of convex polyhedra in

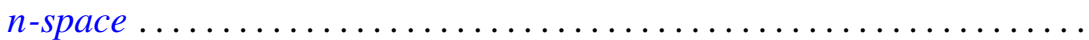

R. H. Bing, Tame Cantor sets in $E^{3}$...

Cecil Edmund Burgess, Collections and sequences of continua in the plane.

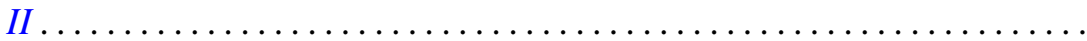

J. H. Case, Another 1-dimensional homogeneous continuum which contains an $\operatorname{arc}$

Lester Eli Dubins, On plane curves with curvature ................. 471

A. M. Duguid, Feasible flows and possible connections .............. 483

Lincoln Kearney Durst, Exceptional real Lucas sequences ................ 489

Gertrude I. Heller, On certain non-linear opeartors and partial differential equations........................................

Calvin Virgil Holmes, Automorphisms of monomial groups

Wu-Chung Hsiang and Wu-Yi Hsiang, Those abelian groups characterized by their completely decomposable subgroups of finite rank ..........

Bert Hubbard, Bounds for eigenvalues of the free and fixed membrane by finite difference methods .........................

D. H. Hyers, Transformations with bounded mth differences. .

Richard Eugene Isaac, Some generalizations of Doeblin's decomposition

John Rolfe Isbell, Uniform neighborhood retracts ..........

Jack Carl Kiefer, On large deviations of the empiric D. F. of vector chance variables and a law of the iterated logarithm...

Marvin Isadore Knopp, Construction of a class of modular functions and forms. II. . .

Gunter Lumer and R. S. Phillips, Dissipative operators in a Banach space....

Nathaniel F. G. Martin, Lebesgue density as a set function ...

Shu-Teh Chen Moy, Generalizations of Shannon-McMillan theorem ...

Lucien W. Neustadt, The moment problem and weak convergence in $L^{2}$

Kenneth Allen Ross, The structure of certain measure algebras...

James F. Smith and P. P. Saworotnow, On some classes of scalar-product algebras.

Dale E. Varberg, On equivalence of Gaussian measures. . 